Chapter 3

\title{
Nanoemulsions - Advances in Formulation, Characterization and Applications in Drug Delivery
}

\author{
S.A. Chime, F.C. Kenechukwu and A.A. Attama \\ Additional information is available at the end of the chapter
}

http://dx.doi.org/10.5772/15371

\section{Introduction}

An ideal drug delivery system fulfils the objective of maximizing therapeutic effect while minimizing toxicity. With the progress in time and advances in science and technology, dosage forms have evolved from simple mixtures and pills, to highly sophisticated systems, which are known as novel drug delivery systems. Nanoemulsions are novel drug delivery systems consisting of emulsified oil and water systems with mean droplet diameters ranging from 50 to $1000 \mathrm{~nm}$. Usually, the average droplet size is between 100 and $500 \mathrm{~nm}$ and can exist as oil-in-water $(\mathrm{o} / \mathrm{w})$ or water-in-oil (w/o) form, where the core of the particle is either oil or water, respectively. Nanoemulsions (Figs. 1 and 2) are made from pharmaceutical surfactants that are generally regarded as safe (GRAS). The surfactant type and concentration in the aqueous phase are chosen to provide good stability against coalescence. Several types of oils-natural semi-synthetic and synthetic are used in the formulation of nanoemulsions. The capacity of nanoemulsions to dissolve large quantities of low soluble drugs along with their mutual compatibility and ability to protect the drugs from hydrolysis and enzymatic degradation make them ideal drug delivery vectors [1]. The major advantages of nanoemulsions as drug delivery carriers include increased drug loading, enhanced drug solubility and bioavailability, reduced patient variability, controlled drug release, and protection from enzymatic degradation [2].

A lot of techniques are available for enhancing absorption of poorly water-soluble drugs, like use of lipid-based systems. Thus enhancement of aqueous solubility in such case is a valuable goal to successfully formulate them into bioavailable dosage forms. A range of novel strategies are currently being developed for efficient delivery of poorly water-soluble drugs, such as the formulation of amorphous solid form, nanoparticles, microemulsions, solid dispersions, melt extrusion, salt formation and formation of water-soluble complexes. Among all, the most 

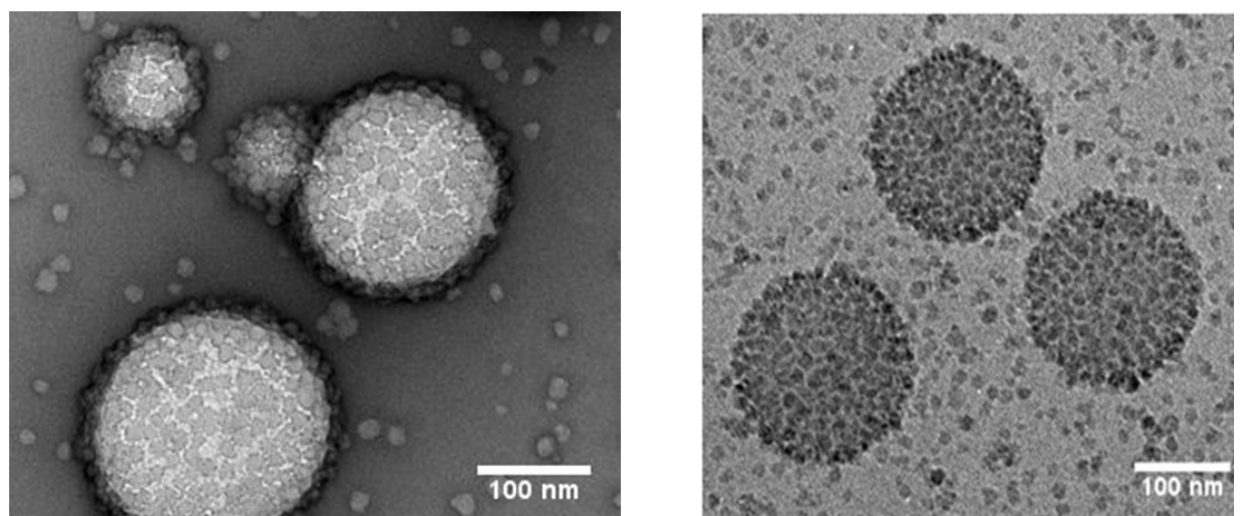

Nanoemulsions courtesy of the Chemical, Materials and Surfaces unit at SP Technical Research Institute of Sweden (former YKI, Institute for Surface Chemistry). Obtained via: http://www.vironova.com/nanoemulsions-casebody. Accessed on April 27, 2014.

Figure 1. A silicate particle-stabilized oil in water nanoemulsion imaged using negative stain TEM (left panel) and cryo TEM (right panel).

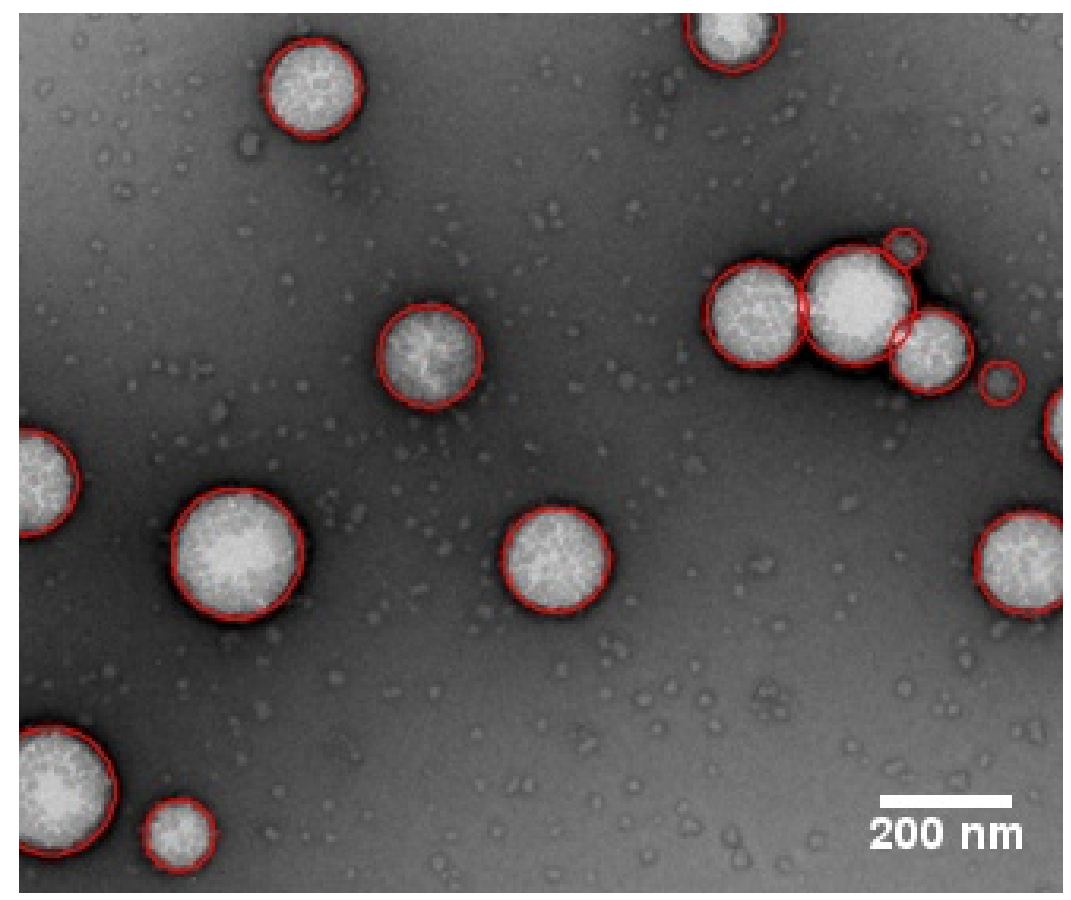

Figure 2. Negative stain TEM image of a silicate particle-stabilized oil in water nanoemulsion. Obtained via: http:// www.vironova.com/nanoemulsions-casebody. Accessed on April 27, 2014. 
accepted approach is the lipid-based formulation approach [3,4]. Lipid-based formulations enhance the absorption by enhancing solubilization, prolonging gastric residence time, stimulating the intestinal lymphatic transport pathway, altering intestinal permeability, reduced activity of efflux transporters and reduced metabolism. Lipid-based formulations present a large range of optional systems such as solutions, suspensions, self-emulsifying systems and nanoemulsions.

Nanoemulsions can be prepared by high- and low-energy methods. Both high-energy and lowenergy methods can produce stable nanoemulsions. High-pressure homogenizer or ultrasound generator can be used for the preparation of nanoemulsion by high-energy emulsification method. Self emulsification and phase inversion methods-phase inversion temperature and phase inversion composition are low-energy methods for the preparation of nanoemulsions. Low-energy emulsification methods depend on the phase behaviour and properties of the constituents, and they utilize the stored energy of the system to form nano droplets. The emulsification can be brought about by changing the parameters such as temperature and composition, which would affect the hydrophilic lipophilic balance of the system.

This chapter focused on recent advances in the formulation, characterization and application of nanoemulsions in drug delivery. Nanoemulsion can be formulated for delivery of drugs through various routes. Nanoemulsions are well tolerated orally and on the skin and mucous membranes when used to deliver topically active drugs. As a result they are used as vehicles for drugs active against herpes labialis, fungal infections, bacterial infections, vaginitis, etc. Nanoemulsion globules can fuse with membranes of lipid-containing organisms facilitating penetration and transfer. Less amount of surfactant is required in nanoemulsions compared to other emulsion systems. This can increase the bioavailability of poorly soluble drugs since small particles easily cross the absorption membrane. Furthermore, very small size provides large surface area which eases the solubilization and penetration through the skin or epithelial layer [5].

\section{Nanoemulsion based delivery system: types and properties}

\subsection{Self emulsifying formulations (SEFs)}

Self-emulsifying formulations (SEFs) are mixtures of oil, surfactant, co-surfactant, and cosolvents (absence of external phase water) and forms a transparent isotropic solution, which emulsify under gentle agitation similar to those which would be encountered in gastro intestinal tract (GIT). It has been recognized that this formulation when administered orally undergo spontaneous emulsification in aqueous GI fluids [6]. This emulsified oil (triglycerides) stimulates bile secretion and drug containing oil droplets are further emulsified by bile salts. Lipid droplets are then metabolized by lipases and co lipases, secreted from the salivary gland, gastric mucosa and pancreas, which also hydrolyze the triglycerides into di-and monoglycerides and free fatty acids. Further, solubilization of these molecules occurs during the passage through the GI tract and eventually forms a range of emulsion droplets, vesicular structures 
and mixed micelles containing bile salts, phospholipids and cholesterol [6]. Upon mixing with water the system SEFs have an ability to form fine colloidal droplets with very high surface area. In many cases, this accelerates the digestion of the lipid formulation, improves absorption, and reduces food effect and inter-subject variability [7]. Self emulsifying formulations distribute readily in the GI tract, the digestive motility of the stomach and the intestines provides sufficient agitation enough for the spontaneous formation of emulsions $[8,9]$.

SEFs prepared using surfactants of $\mathrm{HLB}<12$ possess high stability and improved dissolution (for poorly soluble drugs) due to enhancement in surface area on dispersion [6]. Therefore, their absorption is independent of bile secretion and ensures a rapid transport of poorly soluble drugs into the blood [6].

According to Reiss [10], self emulsification occurs when the entropy changes that favor dispersion is greater than the energy required to increase the surface area of the dispersion [10]. The free energy of the conventional emulsion is a direct function of the energy required to create a new surface between the oil and water phase and can be described by the equation:

$$
\Delta G=\sum N_{i} 4 \pi r_{i}^{2} \sigma
$$

where, $\Delta G$ is the free energy associated with the process, $r_{i}$ is the radius of droplets, $N_{i}$ is the number of droplets, $\sigma$ is the interfacial energy [11]. The two phases of the emulsion tend to separate with time to reduce the interfacial area and thus, minimize the free energy of the system(s). The conventional emulsifying agents stabilize emulsions resulting from aqueous dilution by forming a monolayer around the emulsion droplets, reducing the interfacial energy and forming a barrier to coalescence. On the other hand, emulsification occurs spontaneously with SEDDS, as the free energy required to form the emulsion is low, whether positive or negative [12]. For emulsification to take place, it is vital for the interfacial structure to offer negligible or no resistance against surface shearing [13]. The ease of emulsification has been suggested to be related to the ease of water penetration into various liquid crystals or gel phases formed on the surface of the droplet [14-16]. The interface between the oil and aqueous continuous phases is formed upon addition of a binary mixture (oil/non-ionic surfactant) to water [14]. This is followed by solubilization within the oil phase, as a result of aqueous penetration through the interface. Invariably, this tends to occur until the solubilization limit is attained close to the interphase. Further, aqueous penetration will lead to the formation of the dispersed liquid crystal phase. Ultimately, everything that is in close proximity with the interface will be liquid crystal, the actual amount of which depends upon the emulsifier concentration in the binary mixture. Hence, following gentle agitation of the self-emulsifying system, water rapidly penetrates into the aqueous cores leading to interface disruption and droplet formation [16].

When compared with emulsions, which are sensitive and metastable dispersed forms, SEFs are physically stable formulations that are easy to manufacture. Thus, for lipophilic drugs that exhibit dissolution rate-limited absorption, these systems may offer an improvement in the rate and extent of absorption and result in more reproducible blood time profiles [17]. SEFs 
have been transformed into solid dosage forms using techniques such as melt granulation, where the lipid excipient acts as a binder and solid granules are produced on cooling. Solvents or supercritical fluids can be used with semisolid excipients, which are solubilized and then the solvent evaporated to produce a waxy powder. Spraying techniques can be used to produce powder formulations. These techniques enable the production of granules or powders that can then be compressed into a tablet form or filled into capsules. In all cases, the lipid excipients used must be semi-solid at room temperature [18].

\subsubsection{Self emulsifying drug delivery systems (SEDDS)}

Self-emulsifying drug delivery system (SEDDS) is a strategy that has drawn wide research interest, basically due to its distinct capacity to solubilize and improve the bioavailability of hydrophobic drugs. This it does by ensuring aqueous solubility of the lipophilic drug [19]. The presence of oil makes SEDDS unique and distinguishes them from ordinary surfactant dispersions of drugs [20]. SEDDS are isotropic combination of drug, lipid/oil, cosolvents and surfactants [19]. On dilution by an aqueous phase they form fine stable oil-in-water $(\mathrm{o} / \mathrm{w})$ emulsions or fine lipid droplets which is the characteristic feature of these systems. When such a formulation is released into the lumen of the GIT, it disperses to form a fine emulsion generally o/w emulsion. SEDDS are generally formulated with triglyceride oils and ethoxylated nonionic surfactants. In general, the concentration of surfactant is greater than $25 \%$ in the formulation. The size of droplets ranges approximately less than $100 \mathrm{~nm}$ [19].

SEDDS are believed to be superior compared with lipid solutions due to the presence of surfactants in the formulations leading to a more uniform and reproducible bioavailability [7]. Advantages of SEDDS include more consistent drug absorption, selective targeting of drug(s) toward specific absorption window in GIT, protection of drug(s) from the gut environment, control of delivery profiles, reduced variability including food effects, enhanced oral bioavailability enabling reduction in dose and high drug loading efficiency [21]. Self emulsifying formulations spread readily in the gastrointestinal tract (GIT), and the digestive motility of the stomach and intestine provide the agitation necessary for self emulsification. These systems advantageously present the drug in dissolved form and the small droplet size provides a large interfacial area for the drug absorption. SEDDSs typically produce emulsions with turbid appearance, and droplet size between $200 \mathrm{~nm}$ to $5 \mu \mathrm{m}$ while self micro emulsifying drug delivery systems (SMEDDSs) form translucent micro-emulsions with droplet size of less than $200 \mathrm{~nm}$. However, self nano emulsifying drug delivery systems (SNEDDS) produces clear or transparent emulsion with droplets size less than $100 \mathrm{~nm}[22,23]$. When compared with emulsions, which are sensitive and metastable dispersed forms, SEFs are physically stable formulations that are easy to manufacture. Thus, for lipophilic drug compounds that exhibit dissolution rate-limited absorption, these systems may offer an improvement in the rate and extent of absorption and result in more reproducible blood time profiles [17]. SEDDS are prepared in two forms: Liquid and solid SEDDS (S-SEDDS). S-SEDDS are prepared by solidification of liquid self-emulsifying components into powder. This powder is then used to produce various solid dosage forms, for example self-emulsifying pellets, self-emulsifying tablets etc [19]. S-SEDDS do not suffer with the problems like liquid SEDDS (L-SEDDS). It has 
the advantages like low manufacturing cost, more stability and is more patient compliance, because they are available as solid dosage form in tablets or pellet form. In many studies it have been reported that SEDDS are used for delivering and targeting hydrophobic drugs such as coenzyme Q10, halofantrine, vitamin E and cyclosporine-A [19]. The solid SEDDS focus on the incorporation of liquid/semisolid ingredients into powders employing diverse solidification techniques like spray drying, melt granulation, moulding, melt extrusion, and nanoparticle technology. The powders can then be formulated as solid dosage forms like selfemulsifying tablets and self-emulsifying pellets [16]. Alternative approaches for the development of solid SEDDS comprise adsorption by solid carriers like microcrystalline cellulose, colloidal silica and various viscosity grades of HPMC, and use of high melting point solid excipients like Lutrol ${ }^{\circledast}$ and Gelucire ${ }^{\circledast}$ [16]. The idea of blending the potential SEDDS with that of the pellets through the inclusion of a self-emulsifying mixture into microcrystalline cellulose, and the production of pellets using extrusion-spheronization was first introduced by Newton et al [24].

High levels of surfactant typically present in SEDDS formulations can invariably lead to severe GI side-effects. Hence, a new class of SEDDS formulations, i.e., supersaturable SEDDS (SSEDDS) has been designed to reduce the amount of surfactant by incorporating a water soluble polymeric precipitation inhibitor (PPI) [25] Such formulations have been developed specifically to reduce the surfactant side-effects and achieve rapid absorption of poorly soluble drugs [16]. The system is intended to generate and maintain a metastable supersaturated state in vivo by preventing or minimizing the precipitation of the drug through the use of a suitable PPI. Supersaturation is intended to increase the thermodynamic activity of the drug beyond its solubility limit, and therefore, to result in an increased driving force for transit into and across the biological barrier [26]. The S-SEDDS formulations have been demonstrated to improve both the rate and extent of the oral absorption of poorly water-soluble drugs quite effectively [25, 27, 28]. The inclusion of cellulosic polymers in the S-SEDDS formulation tends to effectively suppress the precipitation of drugs [29]. Various viscosity grades of hydroxypropyl methylcellulose (HPMC) are well-recognized for their ability to inhibit crystallization and, thereby, generate and maintain their supersaturated state for extended time periods [16]. In vitro dilution of the S-SEDDS formulation results in the formation of a microemulsion, followed by slow crystallization of the drug on standing indicating that the supersaturated state of the system is prolonged by HPMC in the formulations. In the absence of HPMC, the SEDDS formulation undergoes rapid precipitation, yielding a lower drug concentration [25]. The significantly reduced amount of surfactant used in the S-SEDDS formulation approach significantly reduces toxicity and improves safety profile over the conventional SEDDS formulations [16].

Positively charged SEDDS have also been produced; many physiological studies have proved that the apical potential of absorptive cells, as well as that of all other cells in the body, is negatively charged with respect to the mucosal solution in the lumen [16]. The drug exposure of the positively charged SEDDS has been found to be higher as well as the conventional formulations especially for bioavailability enhancement. The binding of the cationic SEDDS has been found to be much higher compared with the anionically charg- 
ed formulation, suggesting increased adhesion of the droplets to the cell surface due to electrostatic attraction [16].

Different dosage forms of S-SEDDS include the dry emulsions, self-emulsifying capsules, selfemulsifying sustained/controlled-release tablets, self-emulsifying sustained/controlledrelease pellets, self-emulsifying solid dispersions, self-emulsifying beads, self-emulsifying sustained-release microspheres, self-emulsifying nanoparticles, self-emulsifying suppositories and self-emulsifying implant [29 - 34].

\subsubsection{Advantages of SEDDS}

SEDDS possess the following advantages among others [11]:

- Improvement and reduction in the variability of GI absorption of poorly water soluble, lipophilic drugs.

- Possible reduction in, or elimination of, a number of development and processing steps (e.g., salt selection or identification of a stable crystalline form of the drug, coating, taste masking, and reduced need for containment and clean-up requirements during manufacture of highly-potent or cytotoxic drug products).

- Food does not interfere with the absorption of drug by use of such systems.

- Relative ease of manufacture using readily available equipment.

- The dose ranging from less than $25 \mathrm{mg}$ to greater than $2000 \mathrm{mg}$ can be administered by using these systems.

- These systems enhance oral bioavailability due to bypass of hepatic metabolism and delivers drug directly into systemic circulation.

- Inhibition of p-glycoprotein mediated drug efflux and pre-absorptive metabolism by gut membrane bound cytochrome enzyme.

- Protection of sensitive drug substances

- High drug payloads.

- Liquid or solid dosage forms.

- Reduced energy requirement for emulsion formation.

- Control of delivery profile

- Promotion of lymphatic drug transport.

- They enhance absorption of lipophilic drugs by stimulating pancreatic and biliary secretions and by prolongation of gastric residence time.

\subsubsection{Disadvantages of SEDDS}

Disadvantages of SEDDS include [11, 35]: 
- Lack of good predicative in vitro models for assessment of the formulations.

- Traditional dissolution methods do not work, because formulations are independent on digestion prior to release of the drug.

- In vitro model needs further development and validation.

- Different prototype lipid based formulations needs to be developed and tested in vivo.

- Chemical instabilities of drugs and high surfactant concentrations in formulations (approximately 30-60\%) may irritate GIT.

- Volatile co solvents may migrate into the shells of soft or hard gelatin capsules, resulting in the precipitation of the lipophilic drugs.

- The precipitation tendency of the drug on dilution may be higher due to the dilution effect of the hydrophilic solvent.

- Formulations containing several components become more challenging to validation.

\subsubsection{Self nano emulsifying drug delivery systems (SNEDDSs)}

Self-nano emulsifying drug delivery systems (SNEDDS) are isotropic mixtures of oil, surfactant, co-surfactant and drug that form fine oil-in-water nanoemulsion when introduced into aqueous phases under gentle agitation. SNEDDS spread readily in the gastrointestinal tract, and the digestive motility of the stomach and the intestine provide the agitation necessary for self-emulsification [36]. SEDDSs typically produce emulsions with turbid appearance, and droplet size between $200 \mathrm{~nm}$ to $5 \mu \mathrm{m}$, while self micro emulsifying drug delivery systems (SMEDDSs) form translucent micro-emulsions with droplet size of less than $200 \mathrm{~nm}$. However, self nano-emulsifying drug delivery systems (SNEDDS) produce clear or transparent emulsion with droplets size less than $100 \mathrm{~nm}[22,23]$.

Successful formulation of SNEDDS depends on the thorough understanding of the spontaneous nano-emulsification process and also on the physicochemical and biological properties of the components used for the fabrication of SNEDDS. The factors influencing the phenomenon of self nano-emulsification are:

- The physicochemical nature and concentration of oily phase, surfactant and co-emulsifier or co surfactant or solubilizer (if included)

- The ratio of the components, especially oil-surfactant ratio

- The temperature and $\mathrm{pH}$ of the aqueous phase where nano-emulsification would occur

- Physicochemical properties of the drug, such as hydrophilicity/lipophilicity, pKa and polarity.

These factors should receive attention while formulating SNEDDS. In addition, the acceptability of the SNEDDS components for the desired route of administration is also very important while formulating SNEDDS [36]. 
SNEDDS offer a reduction in bioavailability and can offer reproducibility in plasma profiles of drugs. The ability of the SNEDDS in improving $C_{\max }$ and oral bioavailability or therapeutic effect has been established for various hydrophobic drugs. The improvement in bioavailability can be translated into reduction in the drug dose and dose-related side effects of many hydrophobic drugs, such as antihypertensive and antidiabetic drugs [37]. Transretinol acetate SNEDDS emulsion, anti hyperlipidemic, probucol, estrogen receptor antagonist, tamoxifen citrate, calcium channel blocker, felodipine, and beta blocker, carvedilol have been formulated as SNEDDS [36]. SNEDDS are used for enhancing the solubility of anti-inflammatory drugs such as indomethacin [38]. Fibrinolytic drugs such as simvastatin, atorvastatin, valsartan, gemfibrozil were also formulated as SNEDDS for improved bioavailability. Super-SNEDDS of simvastatin show increased bioavailability compared to the conventional SNEDDS due to the increased drug loading [39]. Solid SNEDDS of valsartan enhanced the bioavailabilty potential due to the presence of porous carriers and also showed stability for about six months which is an important factor [40]. Hormones such as ondasteron hydrochloride and insulin were also delivered orally by using SNEDDS. Solid SNEDDS of ondasteron showed increased bioavailbility than the pure drug [41]. Insulin was formulated into SNEDDS by first forming insulin-phospholipid complex (IPC) and this was used as oil phase in the formulation. This showed good hypoglycemic effect in diabetic Wistar rats for oral administration. Hence IPC can be used for the oral delivery of insulin [42].

Anti-cancer drugs were also formulated as SNEDDS. They include raloxifene hydrochloride, cyclosporine A, paclitaxel, flutamide. In raloxifene, the uptake of the drug by endocrine organs was assessed by administering the SNEDDS in alkalinized and non-alkalinized form to Wistar rats. Non-alkalinized form showed good uptake by the endocrine organs than the alkalinized form [43]. SNEDDS pellets of cyclosporine A were formulated by fluid bed coating technique and this improved the in vivo performance of the drug [44]. The drug release profile of paclitaxel was improved by SNEDDS [45] and the dissolution rate was also faster compared to that of the pure drug in flutamide $[37,46]$.

SNEDDS are given in the form of soft or hard gelatin capsules. They reach the gastro intestinal tract and the GI motility of the stomach provides the agitation for self-emulsification. Because of this self-emulsification the drug is given as small droplets with size less than $5 \mu \mathrm{m}$ for improved solubility. After administering orally, lingual and pancreatic lipases act on the oily phase of the SNEDDS that result in the formation of emulsified mono-glycerides, di-glycerides and fatty acids. This in the presence of bile acids leads to the formation of intestinal mixed micelles. When these mixed micelles pass through the enterocytes, it leads to the formation of chylomicrons. These drain the drug into the lymphatic vessels and not in the blood vessels thus bypassing the first pass effect. Thus the oral bioavailability gets increased [37].

\subsubsection{Advantages of SNEDDS}

Advantages of SNEDDS include [36]:

- Protection of sensitive drug substances.

- Selective targeting of drug(s) toward specific absorption window in GIT. 
- Enhanced oral bioavailability enabling reduction in dose.

- High drug payloads.

- It can be easily stored since it belongs to a thermodynamics stable system.

- Fine oil droplets would pass rapidly and promote wide distribution of the drug throughout the GIT, thereby minimizing the irritation frequently encountered during extended contact between bulk drug substance and the gut wall.

- As compared with oily solutions, they provide a large interfacial area for partitioning of the drug between oil and water [36].

\subsubsection{Disadvantages of SNEDDS}

Disadvantages of SNEDDS include [36]:

- Lack of good predicative in vitro models for assessment of the formulations because traditional dissolution methods do not work, because these formulations potentially are dependent on digestion prior to release of the drug. To mimic this, an in vitro model simulating the digestive processes of the duodenum has been developed.

- Need of different prototype lipid based formulations to be developed and tested in vivo in a suitable animal model

\subsubsection{Factors affecting SNEDDS}

There are many factors that affect SNEDDS viz:

- Drugs which are administered at very high dose are not suitable for SNEDDS, unless they exhibit extremely good solubility in at least one of the components of SNEDDS, preferably lipophillic phase. The drugs exhibit limited solubility in water and lipids are most difficult to deliver by SNEDDS.

- The ability of SNEDDS to maintain the drug in solubilized form is greatly influenced by the solubility of the drug in oily phase. If the surfactant or co-surfactant is contributing to a greater extent for drug solubilization, then there could be a risk of precipitation, as dilution of SNEDDS will lead to lowering of solvent capacity of surfactant or co-surfactant [36].

\subsubsection{Solid self-nanoemulsifying drug delivery systems (SSNEDDSs)}

Solid SNEDDS was developed in order to eliminate the disadvantages associated with liquid SNEDDS handling, manufacturing and stability. Solid SNEDDS in the form of dry, solid powders would help in overcoming the limitations associated with liquid SNEDDS. Solid dosage forms are most stable and are convenient for handling; therefore, attempts are made to convert the liquid systems into solid SNEDDS. Various techniques, such as spray drying, freeze drying and adsorption on carriers, can be employed to convert liquid SNEDDS into solid SNEDDS compressed into tablets. The selection of a particular process for preparation of solid SNEDDS would depend on the content of oily excipient of the formulation, 
properties of active pharmaceutical ingredients, such as solubility, heat stability and compatibility with other ingredients [47].

The simplest technique to convert liquid SNEDDS to solid SNEDDS is by adsorption onto the surface of carriers or by granulation using liquid SNEDDS as a binder. This technique is uncomplicated, cost effective, easily optimized and industrially scalable. It can be used for heat-and moisture-sensitive molecules, thus providing an advantage over other techniques, such as spray drying and freeze drying. Various excipients utilized for the preparation of solid oral dosage forms can be employed for adsorption. The excipients should possess large surface areas to adsorb sticky and sometimes viscous oily SNEDDS formulation [47].

The ability of different excipients, such as dibasic calcium phosphate, lactose, microcrystalline cellulose, colloidal silicon dioxide and Neusilin, to adsorb cefpodoxime proxetil SNEDDS have been studied [47]. Solidification techniques for converting liquid/semisolid SEDDS/SNEDDS to solids include:

- Capsule filling with liquid and semisolid self-emulsifying formulations: Capsule filling is the simplest and the most common technology for the encapsulation of liquid or semi solid SE formulations for the oral route. The advantages of capsule filling are simplicity of manufacturing, suitability for low-dose highly potent drugs and high drug loading up to $50 \%(\mathrm{w} / \mathrm{w})$ potential [48].

- Spray drying: This technique involves the preparation of a formulation by mixing lipids, surfactants, drug, solid carriers, and solubilization of the mixture before spray drying. The solubilized liquid formulation is then atomized into a spray of droplets. The droplets are introduced into a drying chamber, where the volatile phase (e.g. the water contained in an emulsion) evaporates, forming dry particles under controlled temperature and airflow conditions. Such particles can be further prepared into tablets or capsules [48].

- Spray cooling: Spray cooling also referred to as spray congealing is a process whereby the molten formula is sprayed into a cooling chamber. Upon contact with the cooling air, the molten droplets congeal and re-crystallize into spherical solid particles that fall to the bottom of the chamber and subsequently collected as fine powder. The fine powder may then be used for development of solid dosage forms, tablets or direct filling into hard shell capsules. Many types of equipment are available to atomize the liquid mixture and to generate droplets: rotary pressure, two-fluid or ultrasonic atomizers [49, 50].

- Adsorption to solid carriers: SEDDS can be adsorbed at high levels (up to 70\% (w/w)) onto suitable carriers. Solid carriers can be microporous inorganic substances, high surface area colloidal inorganic adsorbent substances, cross-linked polymers or nanoparticle adsorbents (i.g., silica, silicates, magnesium trisilicate, magnesium hydroxide, talcum, crospovidone, cross-linked sodium carboxymethyl cellulose and crosslinked polymethyl methacrylate). The adsorption technique has been successfully applied to gentamicin and erythropoietin with caprylocaproyl polyoxylglycerides (Labrasol) formulations that maintained their bioavailability enhancing effect after adsorption on carriers [51-53]. 
- Melt granulation: Melt granulation or pelletization is a one step-process allowing the transformation of a powder mix (containing the drug) into granules or spheronized pellets. The technique needs high shear mixing in presence of a meltable binder. This is referred to as "pump-on" technique. Alternatively, the binder may be blended with the powder mix in its solid or semi-solid state and allowed to melt (partially or completely) by the heat generated from the friction of particles during high shear mixing referred to as "melt-in" process. The melted binder forms liquid bridges with the powder particles that shape into small agglomerates (granules) which can, by further mixing under controlled conditions transform to spheronized pellets [48].

- Melt extrusion/Extrusion spheronization: It is a solvent-free process that allows high drug loading $(60 \%)$ as well as content uniformity. Applying extrusion-spheronization, SE pellets of diazepam and progesterone and bi-layered cohesive SE pellets have been prepared [54,55].

\section{Advantages, disadvantages and major challenges of nanoemulsions as drug delivery systems}

The advantages of nanoemulsions drug delivery systems include [56-58]:

- The small size of the droplets allows them to deposit uniformly on substrates. Wetting, spreading and penetration may be also enhanced as a result of the low surface tension of the whole system and the low interfacial tension of the o/w droplets.

- The very small droplet size causes a large reduction in the gravity force and the Brownian motion may be sufficient for overcoming gravity. This means that no creaming or sedimentation occurs on storage.

- The small droplet size also prevents any flocculation of the droplets. Weak flocculation is prevented and this enables the system to remain dispersed with no separation. Nanoemulsions are thermodynamically stable system and the stability allows self emulsification of the system

- The small droplets also prevent their coalescence, since these droplets are elastic, surface fluctuations are prevented.

- Nanoemulsions are suitable for efficient delivery of active ingredients through the skin. The large surface area of the emulsion system allows rapid penetration of actives. It is non-toxic and non-irritant so can be easily applied to skin and mucous membranes.

- The transparent nature of the system, their fluidity (at reasonable oil concentrations) as well as the absence of any thickeners may give them a pleasant aesthetic character and skin feel.

- Unlike microemulsions (which require a high surfactant concentration, usually in the region of $20 \%$ and higher), nanoemulsions can be prepared using reasonable surfactant concentration. For a $20 \%$ o/w nanoemulsion, a surfactant concentration in the region of $5-10 \%$ 
may be sufficient. Nanoemulsions are usually formulated with surfactants, which are approved for human consumption (GRAS), they can be taken by enteric route.

- Nanoemulsions can be applied for delivery of fragrance, which may be incorporated in many personal care products. This could also be applied in perfumes, which are desirable to be formulated alcohol free.

- Nanoemulsions may be applied as a substitute for liposomes and vesicles (which are much less stable) and it is possible in some cases to build lamellar liquid crystalline phases around the nanoemulsion droplets.

- Nanoemulsions can be formulated in numerous dosage foam such as creams, liquids, sprays and foams.

- They do not damage healthy human and animal cells, so nanoemulsions are suitable for human and veterinary therapeutic purposes [56].

- Increase the rate of absorption, increases bioavailability and eliminates variability in absorption

- Helps solubilize lipophilic drug and masks unpleasant taste of some drugs

- Various routes like topical, oral and intravenous can be used to deliver the product.

- Better uptake of oil-soluble supplements in cell cultures. Improve growth and vitality of cultured cells. It allows toxicity studies of oil-soluble drugs in cell cultures.

- Nanoemulsions could enhance the stability of chemically unstable compounds by protecting them from oxidative degradation and degradation by light.

- Possibilities of controlled drug release and drug targeting, and the incorporation of a great variety of therapeutic actives.

\subsection{Major challenges}

Although nanoemulsions provide great advantages as a delivery system, however they suffer for some major challenges and limitations which include [56-58]:

- The formulation of nanoemulsions is an expensive process due to size reduction of droplets is very difficult as it required a special kind of instruments and process methods. For example, homogenizer (instrument required for the nanoemulsions formulation) arrangement is an expensive process. More ever micro-fluidization and ultrasonication (manufacturing process) require large amount of financial support.

- One problem associated with nanoemulsion is their stability. Although it is generally accepted that these systems could remain stable even by years, however, due to the small droplet size, it has been reported that the Oswald ripening could damage nanoemulsions, causing their application to be limited. Therefore, in most cases, nanoemulsions are required to be prepared shortly before their use. 
- Use of a large concentration of surfactant and cosurfactant necessary for stabilizing the nano droplets.

- Limited solubility capacity for high melting substances.

- Nanoemulsion stability is influenced by environmental parameters such as temperature and $\mathrm{pH}$.

- Lack of understanding of the mechanism of production of submicron droplets and the role of surfactants and cosurfactants.

- Lack of demonstration of the benefits that can be obtained from using nanoemulsions when compared with the classical macroemulsion systems.

- Lack of understanding of the interfacial chemistry that is involved in production of nanoemulsions.

\section{Formulation of nanoemulsions}

\subsection{Materials used in preparation of nanoemulsions}

Nanoemulsions are prepared using oils, surfactants and co-surfactants and aqueous phase [59]. Oils used in nanoemulsions preparation include Captex 355, Captex 8000, Witepsol, Myritol 318, Isopropyl myristate, Capryol 90, Sefsol-218, triacetin, isopropyl myristate, castor oil, olive oil, etc. Solubility of the drug in the oil phase is an important criterion for the selection of oils. This is particularly important in the case of oral formulation development, as the ability of nanoemulsion to maintain the drug in solubilized form is greatly influenced by the solubility of the drug in the oil phase. While water-in-oil nanoemulsions are better choice for hydrophilic drugs lipophilic drugs are preferably solubilized in oil-in-water nanoemulsions. Drug loading in the formulation is a very critical design factor in the development of nanoemulsions for poorly soluble drugs, which is dependent on the drug solubility in various formulation components. An understanding of factors influencing drug loading capacity while maintaining the capability of the system to undergo monophasic dilution with water and minimizing the tendency for drug precipitation or crystallization in diluted systems is essential to the design of stable and appropriately low-volume nanoemulsion systems for drug delivery applications [60,61]. Edible oils are not frequently useful due to their poor ability to dissolve large amounts of lipophilic drugs. Moreover, formulation of nanoemulsion with oil of low drug solubility would require incorporation of more oil to incorporate the target drug dose, which in turn would require higher surfactant concentration to achieve oil solubilization, which might increase the toxicity of the system. Novel semi-synthetic medium chain derivatives (as amphiphilic compounds) having surfactant properties are progressively and effectively replacing the regular medium chain triglyceride oils $[62,63]$.

Surfactants used for stabilizing nanoemulsions may be non ionic, zwitterionic, cationic and anionic. The surfactants may include Capryol 90, Gelucire 44/14, 50/13, Cremophor RH 40, Imwitor 191, 742, 780 k, 928, 988, Labrafil CS, M, 2125 CS, Lauroglycol 90, PEG MW > 4000, 
Plurol Oleique CC 497, Poloxamer 124 and 188, Softigen 701, 767, Labrasol, Cremophor EL, Tween 20, Tween 60, and Tween 80, etc. Components of nanoemulsion-based systems are associated with toxicity concerns. Large amounts of surfactants may cause gastrointestinal and skin irritation when administered orally and topically, respectively. Therefore, the proper selection of surfactants is essential. Rational use of the minimum concentration of the surfactant in the formulation is advocated. Nonionic surfactants are relatively less toxic than their ionic counterparts and typically have lower critical micelle concentration (CMCs). Also, o/w nanoemulsion dosage forms for oral or parenteral use based on nonionic surfactants are likely to offer in vivo stability [64]. Therefore, proper selection of surfactants is a crucial factor. Another important criterion is the selection of surfactant with proper hydrophile-lipophilebalance (HLB) value. Hydrophilic surfactants and co-surfactants are considered to prefer the interface and to lower the necessary energy to form the nanoemulsions, thereby improving the stability. For instance, the required HLB value to form $\mathrm{o} / \mathrm{w}$ nanoemulsion is greater than 10 [65]. The right blend of low and high HLB surfactants leads to the formation of a stable nanoemulsion upon dilution with water. The type and nature of the surfactant is also an important factor for consideration; nonionic surfactants are usually selected since they are known to be less affected by $\mathrm{pH}$ and changes in ionic strength, are generally regarded as safe, and are biocompatible; ionic surfactants are less commonly used due to toxicological concerns. Solubilization of oil with the surfactant is also an important factor. It is not necessary that the same surfactant that has good solubilizing power for drugs would have equally good affinity for the oil phase. Surfactant-oil miscibility can thus give an initial indication on the possibility of nanoemulsion formation with this system.

Cosurfactants are added to obtain nanoemulsion systems at low surfactant concentration [66]. Short-to medium-chain-length alcohols (C3-C8) are commonly added as cosurfactants, which further reduce the interfacial tension and increase the fluidity of the interface $[67,68]$. They also increase the mobility of the hydrocarbon tail and allow greater penetration of the oil into this region. Alcohols may also increase the miscibility of the aqueous and oily phases due to its partitioning between these phases. Co-surfactants used in nanoemulsions include Transcutol P, glycerin, ethyleneglycol, ethanol, propanol, ethanol, isopropyl alcohol, n-butanol, PEG 400, Carbitol, and propylene glycol. Nanoemulsion area is often used as the assessment criterion for the evaluation of cosurfactants. The larger the size of the nanoemulsion field, the greater the nanoemulsification efficiency of the system.

Moreover, the most important criterion for selection of all the nanoemulsion components is that all the excipients should be pharmaceutically acceptable for oral administration or topical application, etc., depending upon the requirement and falling under GRAS category.

\subsection{Methods of preparation of nanoemulsions}

As nanoemulsions are non-equilibrated systems [69-71], and so their preparation involves the input of a large amount of either energy or surfactants and in some cases a combination of both. As a result, high energy or low energy methods can be used in their formulation [70]. Although high energy emulsification method is traditionally used for the preparation of nanoemulsion formulation but low emulsion emulsification method now create an attraction 
due to their wide application and advantages as a formulation and stability aspects. Generally, energy is usually required in emulsion formulation because the process may be non-spontaneous. The production of nanoemulsions costs more energy than that required to produce macroemulsions. Presence of surfactants help lower the surface tensions between oil and water. Small molecules such as non-ionic surfactants lower surface tension more than polymeric surfactants such as poly (vinyl alcohol). Another important role of the surfactant is its effect on the interfacial dilatational modulus [72]. During emulsification an increase in the interfacial area takes place and this causes a reduction in surface excess. The equilibrium is restored by adsorption of surfactant from the bulk, but this takes time (shorter times occur at higher surfactant activity). Because of the lack or slowness of equilibrium with polymeric surfactants, dilatational modulus will not be the same for expansion and compression of the interface [72]. In practice, surfactant mixtures are used and these have pronounced effects on surface tension and dilatational modulus. Some specific surfactant mixtures give lower surface tension values than either of the two individual components. Polymer-surfactant mixtures may show some synergistic surface activity. An important role of the emulsifier is to prevent shearinduced coalescence during emulsification. The requirement is that the continuous phase has a significant excess of surfactant. This excess enables new surface area of the nano-scale droplets to be rapidly coated during emulsification, thereby inhibiting shear-induced coalescence. This excess is generally in the form of surfactant micelles in the continuous phase. These micelles dissociate into monomers that rapidly adsorb onto the surfaces of newly created droplets [71].

\subsubsection{Low energy methods}

As the name suggests, low-energy emulsification methods require low energy for the fabrication of nanoemulsions. These methods are mainly dependent on modulation of interfacial phenomenon/phase transitions and intrinsic physicochemical properties of the surfactants, coemulsifiers/co-surfactants and oil to yield nano-sized emulsion droplets. The lower energy method, also called the condensation method, is based on the phase transitions taking place during the emulsification process $[73,74]$. These phase transitions result from changes in the spontaneous curvature of the surfactant and can be achieved (i) at constant composition by changing the spontaneous curvature of non-ionic surfactants with temperature, the wellknown Phase Inversion Temperature, PIT, widely used in industry $[75,76]$ or (ii) at constant temperature by varying the composition of the system by the Emulsion Inversion Point (EIP) method [77-79]. In other words, low-energy emulsification method was developed according to the phase behavior and properties of the constituents, to promote the formation of ultrasmall droplets [80,81]. These low-energy techniques include self-emulsification, phase transition and phase inversion temperature methods [82]. The low energy method is interesting because it utilizes the stored energy of the system to form small droplets. This emulsification can be brought about by changing the parameters which would affect the hydrophilic lipophilic balance (HLB) of the system like temperature, composition, etc. [83,84]. The limitations include complexity, precise approach required and use of synthetic surfactants. In a nutshell, the most commonly used low-energy emulsification methods include: 


\subsubsection{Phase Inversion Temperature (PIT) method}

This method employs temperature-dependent solubility of non-ionic surfactants, such as polyethoxylated surfactants, to modify their affinities for water and oil as a function of the temperature. It has been observed that polyethoxylated surfactants tend to become lipophilic on heating owing to dehydration of polyoxyethylene groups. This phenomenon forms a basis of nanoemulsion fabrication using the PIT method. In the PIT method, oil, water and nonionic surfactants are mixed together at room temperature. This mixture typically comprises o/w microemulsions coexisting with excess oil, and the surfactant monolayer exhibits positive curvature. When this macroemulsion is heated gradually, the polyethoxylated surfactant becomes lipophilic and at higher temperatures, the surfactant gets completely solubilized in the oily phase and the initial o/w emulsion undergoes phase inversion to w/o emulsion. The surfactant monolayer has negative curvature at this stage [75]. At an intermediate temperature (also termed HLB temperature), the non-ionic surfactant has similar affinity for aqueous and oily phase, and this ternary system has extremely low interfacial tension (in the order of $10^{-2}$ $10^{-5} \mathrm{mNm}^{-1}$ ) and spontaneous curvature typically reaches zero [83,84]. The ternary system at this stage typically consists of a D-phase bicontinuous microemulsion or a mixture of a Dphase bicontinuous microemulsion and lamellar liquid crystalline phases. It has been observed that nanoemulsions with very small droplet size and polydispersity index can be generated by rapid cooling of the single-phase or multiphase bicontinuous microemulsions maintained at either PIT or a temperature above PIT (transitional-phase inversion) [76]. Nanoemulsions can also be generated by rapidly diluting the single bicontinuous microemulsions with the aqueous or oil phase (catastrophic phase inversion) to obtain either $\mathrm{o} / \mathrm{w}$ nanoemulsion or w/o nanoemulsion. It has been observed that the characteristics of the nanoemulsion are mainly dependent on the structure of the surfactant at HLB temperature (bicontinuous or lamellar) and also on the surfactant/oil ratio. Initially, PIT method was believed to be useful for fabricating o/w nanoemulsions. However, in recent years, the application of the PIT method has been established for fabricating w/o emulsions and nanoemulsions. It is important to note that the use of lipophilic polyethoxylated surfactants and appropriate modifications in the typical PIT protocol are required for obtaining w/o nanoemulsions [82].

\subsubsection{Solvent displacement method}

The solvent displacement method for spontaneous fabrication of nanoemulsion has been adopted from the nano-precipitation method used for polymeric nanoparticles. In this method, oily phase is dissolved in water-miscible organic solvents, such as acetone, ethanol and ethyl methyl ketone. The organic phase is poured into an aqueous phase containing surfactant to yield spontaneous nanoemulsion by rapid diffusion of organic solvent. The organic solvent is removed from the nanoemulsion by a suitable means, such as vacuum evaporation [78-81].

\subsubsection{Phase Inversion Composition Method (Self-nanoemulsification Method)}

This method generates nanoemulsions at room temperature without use of any organic solvent and heat. Forgirani et al. observed that kinetically stable nanoemulsions with small droplet size $(\sim 50 \mathrm{~nm})$ can be generated by the stepwise addition of water into solution of surfactant in 
oil, with gentle stirring and at constant temperature [77]. Although the components used in the aforementioned investigation were not of pharmaceutical grade, the investigation opened doors to design pharmaceutically acceptable nanoemulsions using a similar approach. The spontaneous nano-emulsification has been related to the phase transitions during the emulsification process and involves lamellar liquid crystalline phases or D-type bicontinuous microemulsion during the process [77].

\subsubsection{High energy methods}

High-energy emulsification methods make use of devices that use very high mechanical energy to create nanoemulsions with high kinetic energy. The high-energy method utilizes mechanical devices to create intensely disruptive forces which break up the oil and water phases to form nano-sized droplets. This can be achieved with ultrasonicators, microfluidiser and high pressure homogenisers $[71,85,86]$. Particle size here will depend on the type of instruments employed and their operating conditions like time and temperature along with sample properties and composition [87]. These methods include high-pressure homogenization and ultrasonic emulsification. High-pressure homogenization is the most common method used for the fabrication of nanoemulsions. During high-pressure homogenization, the coarse macroemulsion is passed through a small orifice at an operating pressure in the range of 500 to 5000 psi. During this process, several forces, such as hydraulic shear, intense turbulence and cavitation, act together to yield nanoemulsions with extremely small droplet size. The resultant product can be re-subjected to high-pressure homogenization until nanoemulsion with desired droplet size and polydispersity index is obtained [71]. Micro-fluidization employs a high-pressure positive displacement pump operating at very high pressures, up to 20,000 psi. This pump forces macroemulsion droplets through the interaction chamber consisting of a series of micro-channels. The macroemulsion flowing through the microchannels collides with high velocity on to an impingement area resulting in very fine nanoemulsions. The nanoemulsions with desired size range and dispersity can be obtained by varying the operating pressure and the number of passes through interaction chambers like highpressure homogenization. Ultrasonic emulsification uses a probe that emits ultrasonic waves to disintegrate the macroemulsion by means of cavitation forces. By varying the ultrasonic energy input and time, the nanoemulsions with desired properties can be obtained [85-87]. High-energy emulsification methods can be employed to fabricate both o/w and w/o nanoemulsions. High-pressure homogenization and microfluidization can be used for fabrication of nanoemulsions at laboratory and industrial scale, whereas ultrasonic emulsification is mainly used at laboratory scale. In addition, high-energy methods require sophisticated instruments and extensive energy input, which considerably increases the cost of nanoemulsions fabrication. This is particularly significant in the pharmaceutical sciences [85-87]. High energy methods allow for a greater control of particle size and a large choice of composition, which in turn controls the stability, rheology and colour of the emulsion. Although high-energy emulsification methods yield nanoemulsions with desired properties and have industrial scalability, they may not be suitable for thermolabile drugs such as retinoids and macromolecules, including proteins, enzymes and nucleic acids. Moreover, high energy methods alone normally do not yield oil droplets $(<100 \mathrm{~nm})$. 


\subsection{Formulation factors that affect the stability of nanoemulsions}

Although nanoemulsions enhance the physical as well as chemical stability of drugs, stability of drug product is one of the problems associated with the development of nanoemulsions [88-91]. Stability studies are performed on nanoemulsions by storing them at refrigerator and room temperatures over a number of months. The viscosity, refractive index and droplet size are determined during this period of storage. Insignificant changes in these parameters indicate formulation stability. Accelerated stability studies can also be performed on the nanoemulsions. In this instance, nanoemulsion formulation are kept at accelerated temperatures and samples withdrawn at regular intervals and analyzed for drug content by stability indicating assay methods. The amount of drug degraded and remaining in nanoemulsion formulation is determined at each time interval [92]. Stability of nanoemulsion formulation may be enhanced by controlling factors such as type and concentration of surfactant and cosurfactant, type of oil phase, methods used, process variables and addition of additives [88-93]. Overall nanoemulsion formulation may be considered as effective, safe and patient compliance formulation for the delivery of pharmaceuticals.

Factors to be considered during preparation of nanoemulsion include the following among others [93]:

a. The prime requirement in nanoemulsion production is that an ultra low interfacial tension should be attained at the oil water interface, so surfactants must be carefully chosen.

b. Concentration of surfactant must be high enough to provide the number of surfactant molecules needed to stabilize the nano droplets.

c. The interface must be flexible to promote the formation of nanoemulsion.

\section{Characterization of nanoemulsions}

Characterization of nanoemulsions involves the physical and chemical tests related to oral liquid dosage forms which includes compatibility of the nanoemulsion components, isotropicity of the formulation, assay, uniformity of content, appearance, $\mathrm{pH}$, viscosity, density, conductivity, surface tension, size and zeta potential of the dispersed phase etc. with respect to the effect of the composition on physical parameters [94-104]. Differential scanning calorimetry (DSC) provides information on the interactions of different components and polarization microscopy using crossed polarizers is employed to confirm isotropicity of the formulation [99]. The process of self-nanoemulsification can be evaluated by visual assessment. Its efficiency would be estimated by determining the rate of emulsification and droplet size distribution. Turbidity measurements are carried out to determine the rapid equilibrium reached by the dispersion and reproducibility of this process. The droplet size of the emulsion is a crucial factor in self-nanoemulsification performance because it determines the rate and extent of drug release as well as absorption. Photon correlation spectroscopy (PCS) and light scattering techniques like static light scattering (SLS), dynamic light scattering (DLS) are a useful method for determination of nanoemulsion droplet size [100]. Viscosity, conductivity 
and dielectric methods provide useful information at the macroscopic level. Viscosity measurements for example can indicate the presence of rod-like or worm-like reverse micelles and conductivity measurements provide the means of determining whether a nanoemulsion is oilcontinuous or water-continuous, as well as providing a means of monitoring phase inversion phenomena [99]. Dielectric measurements are a powerful means of probing both the structural and dynamic features of nanoemulsion system. Structural features of nanoemulsions have been studied using self-diffusion nuclear magnetic resonance (SD NMR) and small angle $x$ ray scattering (SAXS). Freeze fracture electron microscopy has also been used to study nanoemulsion structure, however extremely rapid cooling of the sample is required in order to maintain the structure and minimize the possibility of artifacts [101-103]. Nanoemulsion droplet polarity is also a very important factor in characterizing emulsification efficiency. The HLB, chain length and degree of unsaturation of fatty acids, molecular weight of the hydrophilic portion and concentration of the emulsifier have an impact on the polarity of the oil droplets. Polarity represents the affinity of the drug compound for oil and/ or water and the type of forces formed. Rapid release of the drug into the aqueous phase is promoted by the polarity. The charge of the oil droplets of nanoemulsions is another property that should be assessed. Usually it is negative due to the presence of free fatty acids; however, incorporation of a cationic lipid, such as oleylamine at a concentration range of $1-3 \%$, will yield cationic nanoemulsions [104,105].

The following sub-headings could be used to discuss briefly the parameters commonly employed in the assessment of nanoemulsions:

a. Morphology: The morphology of nanoemulsions can be determined by transmission electron microscopy (TEM) and scanning electron microscopy (SEM). SEM gives a threedimensional image of the globules [105]. The samples are examined at suitable accelerating voltage, usually $20 \mathrm{kV}$, at different magnifications. A good analysis of surface morphology of disperse phase in the formulation is obtained through SEM. Image analysis software may be employed to obtain an automatic analysis result of the shape and surface morphology [106]. In TEM, higher resolution images of the disperse phase are obtained. The sample is negatively stained with $1 \%$ aqueous solution of phosphotungstic acid or by dropping $2 \%$ uranyl acetate solution onto a $200 \mu \mathrm{m}$ mesh size Pioloform ${ }^{\mathrm{TM}}$-coated copper grid or a microscopic carbon-coated grid using a micropipette and the sample examined under a transmission electron microscope at appropriate voltage. Qualitative measurements of sizes and size distribution of TEM micrographs can be performed using a digital image processing programme [107]. More sophisticated techniques, such as x-ray or neutron scattering, atomic force microscopy, or cryo-electron microscopy are typically required to explore the structure and behaviour of nanoemulsions [71].

b. Droplet size, polydispersity and zeta potential: Dynamic light scattering (DLS) otherwise called photon correlation spectroscopy (PCS) is used to analyze the fluctuations in the intensity of scattering by droplets/particles due to Brownian motion [108]. Nanoemulsion droplet size, polydispersity and zeta potential can be assessed by PCS using a particle size analyzer. This instrument also measures polydispersity index, which is a measure of the broadness of the size distribution derived from the cumulative analysis of dynamic light 
scattering. The polydispersity index indicates the quality or homogeneity of the dispersion [109]. PCS gives z-average particle diameter. Laser diffraction is another technique for measuring particle size. The fundamental particle size distribution derived by this technique is volume based and is expressed in terms of the volume of equivalent spheres $(D N \%)$ and weighted mean of the volume distribution (mass mean diameter). Since the laser diffraction system is used for this analysis, a rough equivalent of particle polydispersity could be given by two factors/values namely, uniformity (how symmetrical the distribution is around the median point) and span (the width of the distribution). The span value is defined by the expression:

$$
\text { Span }=(D 90 \%-D 10 \%) / D 50 \%
$$

Where $D N \%(N=10 \%, 50 \%, 90 \%)$, means that the volume percentage of particles with diameters up to $D N \%$ equals to $N \%$. The smaller the span value the narrower the particle size distribution.

c. Viscosity: This is carried out using a viscometer. The viscosity of nanoemulsions is a function of the surfactant, water and oil components and their concentrations. Increasing the water content lowers the viscosity, while decreasing the amount of surfactant and cosurfactant increases interfacial tension between water and oil resulting in increased viscosity. Viscosity is very important for stability and efficient drug release. Nanoemulsion carrier formulations are basically oil-in-water and so in addition to being less greasy than water-in-oil formulations, often possess lower apparent viscosities. They are therefore expected to exhibit faster release of active ingredients and wash out easily after application on the skin surface. Various equipment and methods are available for assessment of rheological properties of nanoemulsion carriers. Monitoring of viscosity change is a method of assessing stability of liquid and semi-solid preparations including nanoemulsion formulations [99].

d. In vitro skin permeation: Franz diffusion cell is used to obtain the drug release profile of the nanoemulsion formulation in the case of formulations for transdermal application. The extent or depth of skin penetration by the released content can be visualized by confocal scanning laser microscopy. In vitro drug release can be determined by dispersing an amount of the preparation in the donor compartment of a Franz cell having a membrane as barrier and monitoring the appearance of the encapsulated drug in the receptor compartment, usually containing phosphate buffer saline (PBS, pH 7.4) and stirring on a magnetic stirrer at $100 \mathrm{rpm}$ at $37 \pm 1{ }^{\circ} \mathrm{C}$. Samples $(1 \mathrm{ml})$ of the dispersion are withdrawn from the receptor medium and replaced with an equivalent amount of the medium at definite intervals. The withdrawn sample is then filtered using a 0.22-50 $\mu \mathrm{m}$ filter (e.g., Millipore, USA) and the drug released then analyzed using HPLC or UV-Vis spectroscopy at wavelength of peak absorption of the drug [110]. An alternative and popular method of ex-vivo release study is performed using diffusion cell. The skin is cut from the ear or abdomen and underlying cartilage and fats carefully removed. Appropriate size of skin is cut and placed on the diffusion cell which had earlier been filled with receptor solution. 
Samples of the vesicular preparation are then applied on the dorsal surface of the skin and the instrument started. At intervals, up to $24 \mathrm{~h}$, samples are withdrawn from the receptor medium and replaced with equal amounts of the medium and the withdrawn samples analyzed for the drug permeated using HPLC or UV spectroscopy [111,112]. Semipermeable membrane such as regenerated cellulose could be used in place of skin for in vitro release studies $[113,114]$. The flux $J$, of the drug across the skin or membrane is calculated from the formula:

$$
\mathrm{J}=\mathrm{Ddc} / \mathrm{dx}
$$

Where $\mathrm{D}$ is the diffusion coefficient and is a function of the size, shape and flexibility of the diffusing molecule as well as the membrane resistance, $\mathrm{c}$ is the concentration of the diffusing species, $\mathrm{x}$ is the spatial coordinate [114].

e. In vivo bioavailability/pharmacodynamic studies: In vivo release study otherwise referred to as dermatopharmacokinetics, is carried out by applying or administering the preparation to whole live animal. Blood samples are then withdrawn at intervals, centrifuged and the plasma (deproteinated) analyzed for the drug content using HPLC. Results obtained from in vitro and in vivo studies are extrapolated to reflect bioavailability of the drug formulation. Moreover, the pharmacodynamic properties of nanoemulsion formulations are also assessed depending on the pharmacological properties of the incorporated drug [81-93,95,101].

\section{Applications of nanoemulsions in drug delivery}

Nanoemulsions could be and have been applied in various aspects of drug delivery including: cosmetics and transdermal delivery of drug, cancer therapy, vaccine delivery, prophylactic in bio-terrorism attack, non-toxic disinfectant cleaner, cell culture technology, formulations for improved oral delivery of poorly soluble drug, ocular and otic drug delivery, intranasal drug delivery, parenteral drug delivery and pulmonary delivery of drugs.

\subsection{Applications in cosmetics}

Recently importance of nanoemulsions have become increasing as good vehicles for the controlled delivery of cosmetics and for the optimized dispersion of active ingredients in particular skin layers. Due to their lipophilic interior, nanoemulsions are more suitable for the transport of lipophilic drug than liposomes Similar to liposomes, nanoemulsions supports the skin penetration of active ingredients and thus increases their concentration in the skin. Another advantage is the small-sized droplet with its high surface area permit effective delivery of the active to the skin. More ever, nanoemulsions gain increasing interest due to their own bioactive effects. This may reduce the trans-epidermal water loss (TEWL), suggesting that the barrier function of the skin is strengthened. Nanoemulsions are acceptable in 
cosmetics because there is no chance of creaming, sedimentation, flocculation or coalescence, which is observed within microemulsions. The incorporation of potentially irritating surfactants can be avoided by using high-energy equipment during manufacturing process. PEGfree nanoemulsions for cosmetics has also been developed and formulations exhibited good stability [56-58,115,116].

\subsection{Antimicrobial nanoemulsions}

Antimicrobial nanoemulsions are o/w droplets that range from 200-600 nm. They are made of oil and water and are stabilized by surfactants and alcohol. The nanoemulsions has a broad spectrum of activity against bacteria like E. coli, salmonella, S. aureus; enveloped viruses like HIV, herpes simplex; fungi like candida, dermatophytes, and spores like anthrax. The nanoemulsions particles are thermodynamically driven to fuse with lipid-containing organisms. This fusion is enhanced by the electrostatic attraction between the cationic charge of the emulsion and the anionic charge on the pathogen. When enough nanoparticles fuse with the pathogens, they release part of the energy trapped within the emulsion. Both the active ingredient and the energy released destabilize the pathogen lipid membrane, resulting in cell lysis and death. In the case of spores, additional germination enhancers are added into the emulsion. Once starting of germination takes place, the germinating spores become susceptible to the antimicrobial action of the nanoemulsions. An aspect of the nanoemulsions is their highly selective toxicity to microbes at concentration range that are non-irritating to skin or mucous membrane. The safety range of nanoemulsions is because of the low amount of detergent in each droplet, yet when acting in concert, these droplets have enough energy and surfactant to destabilize targeted microbes without affecting healthy cells. Nanoemulsions can get a level of topical antimicrobial activity, which can only be previously achieved by systemic antibiotics [56-58,115].

\subsection{Prophylactic in bio-terrorism attack}

Because of their antimicrobial activity, research has begun on use of nanoemulsions as a prophylactic medicated dosage form, a human protective treatment, to prevent the people exposed to bio-attack such as Anthrax and Ebola. The broad-spectrum nanoemulsions were checked on surfaces by the US Army (RestOps) in Dec 1999 for decontamination of Anthrax spore. It was checked again by RestOps in March 2001 as a chemical decontamination agent. This technology has been tested on gangrene and clostridium botulism spores, and can even be used on contaminated wounds to salvage limbs. The nanoemulsions can be formulated into a cream, foam, liquid and spray to decontaminate a large number of materials, which is marketed as NANOSTAT ${ }^{\mathrm{TM}}$ (Nanobio Corp.) [56-58,115].

\subsection{Nanoemulsions in vaccines delivery}

This medication delivery system uses nanotechnology to vaccinate against human immunodeficiency virus (HIV). There is recent evidence that HIV can infect the mucosal immune system. Therefore, developing mucosal immunity through the use of nanoemulsions may become very important in the future fight against HIV [50]. The oil-based emulsion is admin- 
istered in the nose, as opposed to traditional vaccine routes. Recent research results indicate that genital mucosa immunity may be attained with vaccines that are administered into the nasal mucosa [56-58,115]. Nanoemulsions are being used to transport inactivated organisms to a mucosal surface to produce an immune response. The first applications as vaccine, an influenza vaccine and an HIV vaccine, can proceed to clinical trials. The nanoemulsion causes proteins applied to the mucosal surface to be adjuvant and it help uptake by antigen presenting cells. This results in the significant systemic and mucosal immune response due to that the production of specific IgG and IgA antibody as well as cellular immunity. Work in influenza has shown that animals can be prevented against influenza after a single mucosal exposure to the virus mixed with thenanoemulsions. Research has also show that animals exposed to recombinant gp120 in nanoemulsions on their nasal mucosa create significant responses to $\mathrm{HIV}$, thus giving a basis to use of this material as an HIV vaccine. Additional research has been ongoing to complete the proof of concept in animal trials for other vaccines including Anthrax and Hepatitis B. The University of Michigan has licensed this technology to NanoBio [56].

\subsection{Nanoemulsions as non-toxic disinfectant cleaner}

Nanemulsions have been employed as a disinfectant cleaner. A nontoxic disinfectant cleaner for use in routine markets that include healthcare, travel, food processing and military applications has been developed by EnviroSystems. They have been found to kill tuberculosis and a large spectrum of viruses, bacteria and fungi within 5 to $10 \mathrm{~min}$ without any of the hazards posed by other categories of disinfectants. The product requires no warning labels. It does not irritate eyes and can be absorbed through the skin, inhaled or swallowed with harmless effects. The disinfectant formulation is made up of nanospheres of oil droplets less than $100 \mu \mathrm{m}$ which are suspended in water to produce a nanoemulsions requiring only small amounts of the active ingredient, parachlorometaxylenol. The nanospheres have surface charges that efficiently penetrate the surface charges on microorganisms' membranes like breaking through an electric fence. Rather than 'drowning' cells, the formulation allows parachlorometaxylenol to target and penetrate cell walls. So parachlorometaxylenol is applicable at concentration ranges I-2 times lower than those of other disinfectants, so there are no toxic effects on human, animals or the environment [56-58,115].

Other microbial disinfectants need large doses of their respective active ingredients to surround pathogen cell wall, which causes microbe to disintegrate, ideally 'drowning' them in the disinfectant solution. The disinfectant is not flammable and so safe to store anywhere and to use in unstable conditions. It is non oxidizing, non acidic and nonionic. It will not corrode plastic, metals or acrylic, so it makes the product ideal for use on equipment and instruments. It is environmentally safe so the economical cost and health risks associated with hazardous chemical disposal are removed. The preparation is a broad-spectrum disinfectant cleaner that can be applied to any hard surface, including equipment, walls, fixtures, counters, and floors. One product can now take the place of many other, decreasing product inventories and saving valuable storage space. Chemical disposal costs can be removed, and disinfection and cleaning costs can be reduced. Marketed as EcoTru TM (EnviroSystems) [56-58,115]. 


\subsection{Nanoemulsions in cell culture technology}

Cell cultures are used for in vitro assays or to produce biological compounds like an antibodies or recombinant proteins. For optimization of cell growth, the culture medium can be supplemented with a large number of molecules or with blood serum. It has been very difficult to provide the media with oil-soluble substances that are available to the cells, and only few amounts of the lipophilic compounds could be absorbed by the cells. Nanoemulsions are a new method for the delivery of oil-soluble substances to human cell cultures. The system is based on a nanoemulsions that is stabilized by phospholipids. This nanoemulsions is transparent and can be passed through $0.1 \mathrm{~mm}$ filters for sterilization. Nanoemulsions oil droplets are very easily taken up by the cells. The encapsulated oil-soluble substances therefore have a high bioavailability to cells in culture.

The advantages of using nanoemulsions in cell culture technology include:

- Better uptake of oil-soluble supplements in cell cultures.

- Improve growth and vitality of cultured cells.

- Allows toxicity studies of oil-soluble drugs in cell cultures [52 - 55].

\subsection{Nanoemulsion formulations for improved oral delivery of poorly soluble drugs}

Nanoemulsions formulation was developed to increase oral bioavailability of hydrophobic drugs. Paclitaxel was selected as a model hydrophobic drug. The o/w nanoemulsions were made with pine nut oil as the internal oil phase, water as the external phase and egg lecithin as the primary emulsifier. Stearylamine and deoxycholic acid were used to give positive and negative charge to the emulsions, respectively. The formulated nanoemulsions had a particle size range of $100-120 \mathrm{~nm}$ and zeta potential ranging from $34 \mathrm{mV}$ to $245 \mathrm{mV}$. After oral administration of nanoemulsions, a significantly higher concentration of paclitaxel was observed in the systemic circulation compare to control aqueous solution. The results of this study suggest that Nanoemulsions are promising novel formulations which can promote the oral bioavailability of hydrophobic drugs [56-58,115].

\subsection{Nanoemulsions in ocular and otic drug delivery}

Ophthalmic drug delivery is one of the most interesting and challenging endeavors facing the pharmaceutical scientist [117]. It is a common knowledge that the application of eye drops as conventional ophthalmic delivery systems results in poor bioavailability and therapeutic response because of lacrimal secretion and nasolacrimal drainage in the eye [118,119]. Most of the drug is drained away from the precorneal area in few minutes. As a result, frequent instillation of concentrated solutions is needed to achieve the desired therapeutic effects [120]. But, by the tear drainage, the main part of the administered drug is transported via the nasolacrimal duct to the gastric intestinal tract where it may be absorbed, sometimes causing side effects [121]. In order to increase the effectiveness of the drug, a dosage form should be chosen which increases the contact time of the drug in the eye. This may then increase the bioavailability, reduce systemic absorption, and reduce the need for frequent administration 
leading to improved patient compliance. Nanoemulsions could be employed to overcome some of these problems. Dilutable nanoemulsions are potent drug delivery vehicles for ophthalmic use due to their numerous advantages as sustained effect and high ability of drug penetration into the deeper layers of the ocular structure and the aqueous humor. Ammar et al. formulated the antiglaucoma drug dorzolamide hydrochloride as ocular nanoemulsion of high therapeutic efficacy and prolonged effect [122]. These nanoemulsions showed acceptable physicochemical properties and exhibited slow drug release. Draize rabbit eye irritation test and histological examination were carried out for those preparations exhibiting superior properties and revealed that they were nonirritant. Biological evaluation of dorzolamide hydrochloride nanoemulsions on normotensive albino rabbits indicated that these products had higher therapeutic efficacy, faster onset of action, and prolonged effect relative to either drug solution or the market product. It was concluded from the study that formulation of dorzolamide hydrochloride in a nanoemulsion form offered a more intensive treatment of glaucoma, a decrease in the number of applications per day, and a better patient compliance compared to conventional eye drops.

\subsection{Nanoemulsions as a vehicle for transdermal delivery}

Drug delivery through the skin to the systemic circulation is convenient for a number of clinical conditions due to which there has been a considerable interest in this area [123,124]. It offers the advantage of steady state controlled drug delivery over extended period of time, with self administration also being possible, which may not be the case with parenteral route. The drug input can be eliminated at any time by the patient just by removing the transdermal patch. Their transparent nature and fluidity, confers on nanoemulsions a pleasant skin feel. An extra advantage is the total absence of gastrointestinal side effects like irritation and bowel ulcers which are invariably associated with oral delivery. Transdermal drug products have been developed for a number of diseases and disorders including cardiovascular conditions, Parkinsons' and Alzheimer diseases, anxiety, depression, etc. However, the fundamental disadvantage which limits the use of this mode of administration is the barrier imposed by the skin for effective penetration of the bioactives. The three routes by which drugs can primarily penetrate the skin are through the hair follicles, sweat ducts or directly across stratum corneum, which restricts their absorption to a large extent and limits their bioavailability. For improved drug pharmacokinetics and targeting, the primary skin barriers need to be overcome. Also the locally applied drug redistribution through cutaneous blood and lymph vessel system needs to be controlled. Nano sized emulsions are able to easily penetrate the pores of the skin and reach the systemic circulation thus getting channelized for effective delivery [69]. Caffeine has been used for treatment of different types of cancer by oral delivery. Water-in-oil nanoemulsion formulations of caffeine have been developed for transdermal drug delivery. Comparison of in vitro skin permeation profile between these and aqueous caffeine solutions showed significant increase in permeability parameters for the nanoemulsion loaded drugs [125]. Use of nanoemulsions in transdermal drug delivery represents an important area of research in drug delivery, which enhances the therapeutic efficacy and also the bioavailability of the drugs without any adverse effects. It is also regarded as a promising technique with many advantages including high storage stability, low preparation cost, thermodynamic stability, absence of 
organic solvents, and good production feasibility. They have also made the plasma concentration profiles and bioavailability of drugs reproducible. These systems are being used currently to provide dermal and surface effects, and for deeper skin penetration [69]. Many studies have shown that nanoemulsion formulations possess improved trans-dermal and dermal delivery properties in vitro [66,126-133], as well as in vivo [134-136]. Nanoemulsions have improved transdermal permeation of many drugs over the conventional topical formulations such as emulsions and gels [137-141].

Barakat et al prepared nanoemulsions by the spontaneous emulsification method for transdermal delivery of indomathacin [142]. A significant increase in the permeability parameters such as steady-state flux, permeability coefficient, and enhancement ratio was observed in nanoemulsion formulations compared with the conventional indomethacin gel. The antiinflammatory effects of nanoemulsion formulations showed a significant increase in percent inhibition value after 4 hours when compared with conventional indomethacin gel on carrageenan-induced paw edema in rats. Significant increase in permeability parameters was observed in nanoemulsion formulations $(P<0.05)$. The steady-state flux and permeability coefficient for optimized nanoemulsion formulation (were found to be $22.61 \pm 3.45 \mu \mathrm{g} / \mathrm{cm}^{2} / \mathrm{h}$ and $0.22 \times 10^{-2} \pm 0.0003 \mathrm{~cm} / \mathrm{h}$, respectively), which were significant compared with conventional indomethacin gel $(P<0.001)$. Enhancement ratio was found to be 8.939 in optimized formulation compared with indomethacin gel. These results suggested that nanoemulsions can be used as potential vehicles for improved transdermal delivery of indomethacin as an approach to eliminate the side effect of the oral dose.

Singh et al developed nanoemulsion formulation for transdermal delivery of carvedilol to enhance the water solubility as well as bioavailability of drug [143]. O/W nanoemulsions were prepared by the spontaneous emulsification method. Post application plasma carvedilol was increased 6.41 fold to marketed dosage form. The study suggested that nanoemulsion significantly enhanced bioavailability of transdermally applied carvedilol and eliminated the first pass metabolism.

Sajid et al prepared betamethasone valerate nanoemulsions by aqueous phase titration method, using Sefsol, Tween 20, Transcutol P, and distilled water as the oil phase, surfactant, co surfactant and aqueous phase, respectively and evaluated them based on the induction of contact dermatitis in rats using a dispersion of nickel sulfate in solid vaseline at $5 \%$, carrageenan induce inflammation and their irritation study [144]. The optimized nanoemulsion was converted into hydrogel using Carbopol 934. Drug deposition in skin was found to be 58.46 $\mu \mathrm{g} / \mathrm{cm} 2$. In vivo anti-inflammatory activity indicated $84.2 \%$ and $45.05 \%$ inhibition of inflammation in case of developed nanoemulsion gel and marketed cream, respectively. The irritation score was found to be 1.83 which indicates that the optimized nanoemulsion did not cause any irritation. Results of nickel induced dermatitis demonstrate that the nanoemulsion formulation gel did not appear to stimulate an inflammatory or immune response using the contact dermatitis model.

Zhou et al. carried out a study to establish a lecithin nanoemulsion without any synthetic surfactant as a topical delivery vehicle and to evaluate its topical delivery potential [145]. Experimental results demonstrated that an increasing concentration of soybean lecithin and 
glycerol resulted in a smaller size lecithin nanoemulsion droplet and increasing viscosity, respectively. Lecithin nanoemulsion, incorporated into o/w cream, improved the skin hydration capacity of the cream significantly with about 2.5-fold increase when the concentration of lecithin nanoemulsion reached $10 \%$. Lecithin nanoemulsion was also demonstrated to improve the penetrability of Nile red dye into the dermis layer, when an o/w cream, incorporated with Nile-red-loaded lecithin nanoemulsion, applied on the abdominal skin of rat in vivo. Specifically, the arbitrary unit of fluorescence in the dermis layer that had received the cream with a Nile red-loaded lecithin nanoemulsion was about 9.9-fold higher than the cream with a Nile red-loaded general emulsion. These observations suggest that lecithin nanoemulsion could be used as a promising topical delivery vehicle for lipophilic compounds.

Modi et al investigated the potential of a nanoemulsion formulation for topical delivery of aceclofenac [146]. The in vitro skin permeation profile of optimized formulations was compared with that of aceclofenac conventional gel and nanoemulsion gel. A significant increase in permeability parameters such as steady-state flux, permeability coefficient and enhancement ratio was observed in optimized nanoemulsion formulation consisting of $2 \% \mathrm{w} / \mathrm{w}$ of aceclofenac, $10 \% \mathrm{w} / \mathrm{w}$ of Labrafac, $45 \% \mathrm{w} / \mathrm{w}$ surfactant mixture (Cremophor ${ }^{\circledR}$ EL: Ethanol), and 43 $\% \mathrm{w} / \mathrm{w}$ of distilled water. The anti-inflammatory effects of formulation showed a significant increased percent inhibition value after 24 hours when compared with aceclofenac conventional gel and nanoemulsion gel on carrageenan-induced paw edema in rats. These results suggested that nanoemulsions are potential vehicles for improved transdermal delivery of aceclofenac.

Batoota et al investigated the potential of nanoemulsion formulations for transdermal delivery of celecoxib [91]. The in vitro skin permeation profile of optimized formulations was compared with celecoxib gel and nanoemulsion gel. Significant increase in the steady state flux, permeability coefficient and enhancement ratio was observed in nanoemulsion formulations $(p<$ 0.05). The highest value of these permeability parameters was obtained in the formulation that consisted of $2 \%(\mathrm{w} / \mathrm{w})$ of celecoxib, $10 \%(\mathrm{w} / \mathrm{w})$ of oil phase (Sefsol 218 and Triacetin), $50 \%$ $(\mathrm{w} / \mathrm{w})$ of surfactant mixture (Tween-80 and Transcutol-P) and $40 \%(\mathrm{w} / \mathrm{w})$ water. The antiinflammatory effects of the formulation showed a significant increase $(p<0.05)$ in inhibition after 24 hours compared to celecoxib gel and nanoemulsion gel on carrageenan-induced paw edema in rats. These results suggested that nanoemulsions are potential vehicles for improved transdermal delivery of celecoxib.

Harwansh et al evaluated an isotropic and thermodynamically stable nanoemulsion formulation for transdermal delivery of glycyrrhizin, with minimum surfactant and cosurfactant concentrations that could improve its solubility, permeation enhancement, and stability [147]. A significant increase in permeability parameters such as steady-state flux and permeability coefficient was observed in the optimized nanoemulsion formulation, which consisted of $1 \%$ w/w of mono ammonium glycyrrhizinate, 32.4 \% Span 80, $3.7 \%$ Brij 35, $10 \%$ isopropyl alcohol, $46.5 \%$ soyabean oil and $6.4 \%$ distilled water. No obvious skin irritation was observed for the studied nanoemulsion formulation or the gel. The results indicated that nanoemulsions are promising vehicles for transdermal delivery of glycyrrhizin through human cadaver skin, 
without the use of additional permeation enhancers, because excipients of nanoemulsions act as permeation enhancers themselves.

Inayat et al. developed a potential of nanoemulsion formulation for transdermal delivery of tamoxifene citrate for breast cancer [148]. Transdermal permeation of tamoxifene citrate through rat skin was determined by Keshary-Chien diffusion cell. A significant increase in permeability parameter such as steady-state flux was observed in optimized nanoemulsion formulation, which consist of $5 \% \mathrm{w} / \mathrm{w}$ of drug, $4.12 \% \mathrm{w} / \mathrm{w}$ of oil phase, $37.15 \% \mathrm{w} / \mathrm{w}$ of surfactant (mix) and $58.73 \% \mathrm{w} / \mathrm{w}$ of distilled water. It possessed a mean globule size of $68 \mathrm{~nm}$. Transmission electron microscopy demonstrated spherical particle morphology and DSC and FTIR study revealed the compatibility among the ingredient. These results proposed that the prepared system could be promising to improve transdermal efficacy of the tamoxifen citrate.

Shakeel et al investigated the potential of a nanoemulsion formulation for transdermal delivery of aceclofenac [149]. Transdermal permeation of aceclofenac through rat abdominal skin was determined by Franz diffusion cell. The in vitro skin permeation profile of optimized formulations was compared with that of aceclofenac conventional gel and nanoemulsion gel. A significant increase in permeability parameters such as steady-state flux, permeability coefficient, and enhancement ratio was observed in optimized nanoemulsion formulation, which consisted of $2 \% \mathrm{w} / \mathrm{w}$ of aceclofenac, $10 \% \mathrm{w} / \mathrm{w}$ of Labrafil R, $5 \% \mathrm{w} / \mathrm{w}$ of Triacetin R, $35.33 \% \mathrm{w} / \mathrm{w}$ of Tween $80 \mathrm{R}, 17.66 \% \mathrm{w} / \mathrm{w}$ of Transcutol PR, and $32 \% \mathrm{w} / \mathrm{w}$ of distilled water. The anti-inflammatory effects of optimized formulation showed a significant increase in percent inhibition value after 24 hours when compared with aceclofenac conventional gel and nanoemulsion gel on carrageenan-induced paw edema in rats. These results suggested that nanoemulsions are potential vehicles for improved transdermal delivery of aceclofenac.

Shakeel et al presented an overview of the efforts that have been made in the last decade by various researchers in exploring new types of nanoemulsion-based drug delivery system for dermal and transdermal delivery of many hydrophobic compounds [150]. This area of research would be very advantageous for formulation scientists in order to develop some nanoemulsion-based formulations for their commercial exploitation and clinical applications. Moreover, Harwansh et al reviewed efforts made by various researchers in the delivery of phytopharmaceuticals using nanoemulsions [151].

\subsection{Nanoemulsion in cancer therapy and in targeted drug delivery}

Another interesting application, which is experiencing an active development, is the use of nanoemulsion formulations, for controlled drug delivery and targeting [82]. Because of their submicron size, they can easily be targeted to the tumor area. Although nanoemulsions are chiefly seen as vehicles for administering aqueous insoluble drugs, they have more recently received increasing attention as colloidal carriers for targeted delivery of various anticancer drugs, photosensitizers, neutron capture therapy agents, or diagnostic agents. The development of magnetic nanoemulsions is an innovative approach for cancer therapy. These can deliver photosensitizers like Foscan ${ }^{\circledR}$ to deep tissue layers across the skin thereby inducing hyperthermia for subsequent free radical generation. This methodology can be used for the treatment of cancer in the form of photodynamic therapy [152]. 


\subsection{Nanoemulsions and intranasal drug delivery}

Intranasal drug delivery system has now been recognized as a reliable route for the administration of drugs next to parenteral and oral routes. Nasal mucosa has emerged as a therapeutically viable channel for the administration of systemic drugs and also appears to be a favourable way to overcome the obstacles for the direct entry of drugs to the target site [153]. This route is also painless, non-invasive and well tolerated. The nasal cavity is one of the most efficient sites because of its reduced enzymatic activity, high availability of immunoactive sites and its moderately permeable epithelium [154]. There are several problems associated with targeting drugs to brain, especially the hydrophilic ones and those of high molecular weight. This is because of the impervious nature of the endothelium, which divides the systemic circulation and barrier between the blood and brain [155]. The olfactory region of the nasal mucosa provides a direct connection between the nose and brain, and by the use of nanoemulsions loaded with drugs, conditions such as Alzheimer's disease, migraine, depression, schizophrenia, Parkinson's diseases, meningitis, etc. can be treated $[156,157]$. Preparation of nanoemulsions containing risperidone for its delivery to the brain via nose has been reported [158]. It is inferred that this emulsion is more effective through the nasal rather than intravenous route. Another application of intranasal drug delivery system in therapeutics is their use in development of vaccines. Immunity is achieved by the administration of mucosal antigen. Currently, the first intranasal vaccine has been marketed [158]. Among the possible delivery systems, the use of nano based carriers hold a great promise to protect the biomolecules, promote nanocarrier interaction with mucosae and to direct antigen to the lymphoid tissues. Therefore the use of nanoemulsions in intranasal drug delivery system is set to bring about significant results in targeting drugs to the brain in treatment of diseases related to the central nervous system [159]. Bhanushali et al developed intranasal nanoemulsion and gel formulations for rizatriptan benzoate for prolonged action [160]. Various mucoadhesive agents were tried out to form thermo-triggered mucoadhesive nanoemulsions. Mucoadhesive gel formulations of rizatriptan were prepared using different ratios of HPMC and Carbopol 980. Comparative evaluation of intranasal nanoemulsions and intranasal mucoadhesive gels indicated that greater brain-targeting could be achieved with nanoemulsions. Other drugs which have been formulated for nasal delivery are insulin and testosterone [161].

\subsection{Nanoemulsions and parenteral drug delivery}

This is one of the most common and effective routes of drug administration usually adopted for actives with low bioavailability and narrow therapeutic index. Their capacity to dissolve large quantities of hydrophobics, together with their mutual compatibility and ability to protect the drugs from hydrolysis and enzymatic degradation make nanoemulsions ideal vehicles for the purpose of parenteral transport. Further, the frequency and dosage of injections can be reduced throughout the drug therapy period as these emulsions guarantee the release of drugs in a sustained and controlled mode over long periods of time. Additionally, the lack of flocculation, sedimentation and creaming, combined with a large surface area and free energy, offer obvious advantages over emulsions of larger particle size, for this route of administration [69]. Their very large interfacial area positively influences the drug transport 
and their delivery, along with targeting them to specific sites. Major clinical and pre-clinical trials have hence been carried out with parenteral nanoemulsion based carriers. The advances in these novel drug delivery systems have been reviewed by Patel and Patel [162]. Nanoemulsions loaded with thalidomide have been synthesized where a dose as low as $25 \mathrm{mg}$ leads to plasma concentrations which can be therapeutic [163]. However, a significant decrease in the drug content of the nanoemulsion was observed at $0.01 \%$ drug formulation after two months storage which could be overcome by the addition of polysorbate 80 . Chlorambucil, a lipophilic anticancer agent has been used against breast and ovarian cancer. Its pharmacokinetics and anticancer activity has been studied by loading it in parenteral emulsions prepared by high energy ultrasonication method. Treatment of colon adenocarcinoma in the mouse with this nanoemulsion leads to higher tumor suppression rate compared to plain drug solution treatment, concluding that the drug loaded emulsion could be an effective carrier for its delivery in cancer treatment [164]. Carbamazepine, a widely used anticonvulsant drug had no parenteral treatment available for patients due to its poor water solubility. Kelmann et al. have developed a nanoemulsion for its intravenous delivery, which showed favorable in vitro release kinetics [165]. Parenteral nanoemulsion formulations of the following drugs have been documented as well: diazepam, propofol, dexamethasone, etomidate, flurbiprofen and prostaglandin E1 [166].

The high lipophilicity of diazepam (an anxiolytic and sedative) makes the use of solvents (such as propylene glycol phenyl carbinol and ethanol) for the dissolution of the drug in conventional aqueous preparations (Valium ${ }^{\circledR}$ and Stesolid ${ }^{\circledR}$ ) necessary, leading to pain and thrombophlebitis on the patient during the injection. The development of a nanoemulsion, commercially available under the name of Diazemuls ${ }^{\circledR}$ (Kabi-Pharmacia) allows for the reduction of these adverse effects, keeping stages of distribution and elimination similar to Valium ${ }^{\circledR}$. However, higher doses of Diazemuls ${ }^{\circledR}$ are necessary to obtain the same effect as Valium ${ }^{\circledR}$ since this leads to higher free fraction of plasma diazepam $[167,168]$.

The solution for intravenous administration of etomidate (hypnotic short) due to stability problems, its composition contains $35 \%$ propylene glycol (Hypnomidate ${ }^{\circledR)}[169,170]$. Due to the presence of high osmolarity of the solvent, the administration is associated with various adverse effects such as hemolysis, thrombosis, thrombophlebitis and pain at the site of application [171,172]. A nanoemulsion containing $2 \mathrm{mg} / \mathrm{ml}$ Lipofundin ${ }^{\circledR}$ etomidate in medium chain triglyceride named Lipuro-etomidate ${ }^{\circledR}$ (B. Braun) was developed [173]. The emulsion allowed the reduction of the hemolytic and venous sequelae, besides the pain at the time of application [169-171].

The pharmacokinetics and pharmacodynamics of propofol (anesthetic) are complex. It has an initial rapid distribution of about 2-3 minutes, with high variability between patients and reduced concentrations to subtherapeutic levels within minutes. However, due to its high lipophilicity, it has a high volume of distribution and its complete elimination from the body can take days [174]. Due to the occurrence of anaphylactic effects associated with Cremophor EL, present in the original formulation of propofol nanoemulsion as vehicle for this drugcontaining composition in soybean oil, glycerol, egg yolk lecithin and disodium edentate, this vehicle helped to reduce the volume of distribution of the drug, accelerating their processes of 
clearance by the responsible agencies. This formulation also allowed the use of minimal effective dose need to produce the needed therapeutic effect, allowing a rapid onset and recovery from anesthesia, when compared to a non-lipid (ethanol) solution, thereby generating greater security administration, due to the lower continuous accumulation of the drug, and eliminating the need for constant adjustment of the dose. This product was approved in 1989 in the United States, under the name of Diprivan ${ }^{\circledR} 1$ or $2 \%$ (AstraZeneca / APP Pharmaceuticals) [175]. In Brazil, the product is available as Lipuro 1\% (B. Braun) and Diprivan ${ }^{\circledR} 1$ and $2 \%$ (AstraZeneca), besides the generic 1\% (Eurofarma Labs.) [176]. The various generic formulations currently available are constituted by an additional factor of variability in response between individuals in the induction of anesthesia, apart from the pharmacokinetic characteristics of the drug itself [174] and the differences in lipoprotein profile of each patient, due to the high binding of propofol to low density lipoprotein and albumin [177]. Due to related pain at the injection site and increased triglyceride levels after administration for long periods, some changes in the formulation of Diprivan $^{\circledR}$ adverse effects have been proposed, including some already being marketed as Propofol ${ }^{\circledR}$ Lipuro (B. Braun) as oil core which contains a mixture of oils [178]. The addition of more oil to the formulation allowed the reduction of pain on injection due to increased incorporation of the drug in the oily core and the lower amount of free propofol phase the external aqueous emulsion [171,177-180]. Alternative formulations have been developed, for example, the incorporation of higher concentrations of propofol $(6 \%)$ in the nanoemulsion [180-182], or the development of a propofol prodrug in solution (Aquavan ${ }^{\circledR}$ ) [183].

Furthermore, despite the excellent anti-inflammatory activity of dexamethasone, the clinical use of corticosteroids is limited by numerous side effects $[184,185]$. To circumvent these drawbacks, lipophilic prodrugs in the body that are gradually hydrolyzed to the active metabolite can be used (thus presenting prolonged anti-inflammatory effect). The advantage is the use of lower doses than those used in conventional water soluble form (dexamethasone phosphate), reducing the risks of adverse effects. Considering that nanoemulsions are picked up by inflammatory cells of the mononuclear phagocytic system, nanoemulsions were used as a vehicle for lipophilic prodrug of dexamethasone (palmitate), which is commercially available as Limethason ${ }^{\circledR}$ (Green Cross Co./Mitsubishi Tanabe Pharma Co.). Limethason ${ }^{\circledR}$ showed excellent results in the treatment of rheumatoid arthritis, West syndrome, inflammatory diseases and other autoimmune diseases. While the solution of dexamethasone phosphate is rapidly distributed in water-rich tissues, such as muscles, the nanoemulsion is accumulated mainly in tissues inflamed organs such as liver and spleen. The biodistribution profile is different even if the elimination pattern is similar between the two. Limethason ${ }^{\circledR}$ removes over $80 \%$ of the phagocytic activity of macrophages at a concentration of $0.03 \mathrm{mg} / \mathrm{mL}$ [185].

Flurbiprofen (non-steroidal anti-inflammatory oral use), a lipophilic drug, is used to treat rheumatoid arthritis and other inflammatory diseases associated or not with cancer [186]. The non-availability of oral and/or various gastrointestinal effects caused by this drug often require the use of parenteral route. Considering the severe local irritation caused by the sodium salt of flurbiprofen, it was developed as a prodrug of flurbiprofen (cefuroxime) and because of the lipophilicity of the latter especially in soybean oil, it was incorporated in nanoemulsions for parenteral use (Ropion ${ }^{\circledR,}$ Kaken Pharmaceuticals Co., Lipfen ${ }^{\circledR,}$ GreenCross Co.), and is com- 
mercially available in the Japanese market since 1992. Administration of Ropion ${ }^{\circledR}$ resulted in an increase in area under the concentration-time curve and reduced clearance when compared to the solution. The incorporation of the drug into nanoemulsions containing unesterified ethyl oleate, lecithin and modified egg yolk led to a lower drug accumulation in organs such as the liver and spleen due to the lower uptake by the mononuclear phagocyte system [166].

Prostaglandin E1, which is synthesized in several places of the body, is responsible for various physiological effects such as vasodilatation, lowering of blood pressure, angiogenesis and inhibition of platelet aggregation $[187,188]$. When administered for the treatment of various diseases, it has a short half-life; high doses are needed, leading to numerous adverse effects such as hypotension, diarrhea, local irritation and pain [187]. In this context, nanoemulsions were made commercially available in 1975, PGE1 complexed to cyclodextrins and, in 1985, prostaglandin E1 incorporated in lipid nanoemulsions (Liple ${ }^{\circledR,}$ Mitsubishi Tanabe Pharma Corporation, Palux $^{\circledast,}$ Taisho Pharmaceutical) [189]. Lipid formulations are used to treat cardiovascular diseases because they accumulate in the walls of injured vessels, transporting the drug to the site of vascular injury and to protect it from rapid inactivation by the lungs [187-190].

\subsection{Nanoemulsions and pulmonary drug delivery}

The lung is an attractive target for drug delivery due to noninvasive administration via inhalation aerosols, avoidance of first-pass metabolism, direct delivery to the site of action for the treatment of respiratory diseases, and the availability of a huge surface area for local drug action and systemic absorption of drug. Colloidal carriers (ie, nanocarrier systems) in pulmonary drug delivery offer many advantages such as the potential to achieve relatively uniform distribution of drug dose among the alveoli, achievement of improved solubility of the drug from its own aqueous solubility, a sustained drug release which consequently reduces dosing frequency, improves patient compliance, decreases incidence of side effects, and the potential of drug internalization by cells [191]. Until now, the submicron emulsion system has not yet been fully exploited for pulmonary drug delivery and very little has been published in this area [191]. Bivas-Benita et al. reported that cationic submicron emulsions are promising carriers for deoxyribonucleic acid vaccines to the lung since they are able to transfect pulmonary epithelial cells, which possibly induce cross priming of antigen-presenting cells and directly activate dendritic cells, resulting in stimulation of antigen-specific T-cells [192]. Therefore the nebulization of submicron emulsions will be a new and upcoming research area. However, extensive studies are required for the successful formulation of inhalable submicron emulsions due to possible adverse effects of surfactants and oils on lung alveoli function (adverse interactions with lung surfactant). A novel pressurized aerosol system has been devised for the pulmonary delivery of salbutamol using lecithin-stabilized microemulsions formulated in trichlorotrifluoroethane [193].

\subsection{Nanoemulsions as gene delivery vector}

Emulsion systems have been introduced as alternative gene transfer vectors to liposomes [194]. Other emulsion studies for gene delivery (non-pulmonary route) have shown that binding of the emulsion/DNA complex was stronger than liposomal carriers [195]. This stable emulsion 
system delivered genes more efficiently than liposomes [196]. Silva et al evaluated factors that influence DNA compaction in cationic lipid nanoemulsions [cationic nanoemulsions containing stearylamine (a cationic lipid that presents a primary amine group when in solution, is able to compact genetic material by electrostatic interactions, and in dispersed systems such as nanoemulsions this lipid anchors on the oil/water interface conferring a positive charge to them)] [197]. The influence of the stearylamine incorporation phase (water or oil), time of complexation, and different incubation temperatures were studied. The complexation rate was assessed by electrophoresis migration on agarose gel $0.7 \%$, and nanoemulsion and lipoplex characterization was done by dynamic light scattering (DLS). The results demonstrate that the best DNA compaction process occurs after $120 \mathrm{~min}$ of complexation, at low temperature $(4 \pm$ $1^{\circ} \mathrm{C}$ ), and after incorporation of the cationic lipid into the aqueous phase. Although the zeta potential of lipoplexes was lower than the results found for basic nanoemulsions, the granulometry did not change. Moreover, it was demonstrated that lipoplexes are suitable vehicles for gene delivery.

\section{Nanoemulsions for phytopharmaceuticals}

Recently, considerable attention has been focused on the development of novel drug delivery systems for herbal drugs [198]. However some limitations of plant bioactives like instability in highly acidic $\mathrm{pH}$ and liver metabolism led to drug levels below therapeutic concentration in the blood resulting in less or no therapeutic effect [199]. Hence, encapsulation of plant extracts or its bioactives would minimize their degradation or presystemic metabolism, and serious side effects due to accumulation of drugs to the non-targeted areas and improves the ease of administration in the pediatric and geriatric patients [200]. Lipid nanoemulsions containing oil from medicinal plants or hydrophobic drugs have been shown to improve drug solubility, reduce side effects of various potent drugs, increase the bioavailability of drugs, and to prolong the pharmacological effects in comparison to conventional formulations such as conventional emulsions [201]. Formulation of nanoemulsions containing phytoactives have been reported.

The effect of nanoemulsion on intestinal absorption of colchicine was demonstrated in vivo. Colchicine nanoemulsion was prepared with isopropyl myristate, eugenol, Tween 80, ethanol and water, with eugenol being the oil phase in the formulation. Result obtained indicated that the intestinal absorption of colchicine was significantly enhanced by the nanoemulsion formulation [202]. Genistein has been shown to possess anticancer activities in different experimental systems, yet the same effects could not be translated in the clinical setting due to its poor bioavailability. Researcher have tried various nano approaches including incorporation of genistein into topical nanoemulsion formulations composed of egg lecithin, medium chain triglycerides or octyldodecanol and water by spontaneous emulsification with improved activity [203]. Oil in water nanoemulsion formulation has also demonstrated increased antiinflammatory activity of curcumin [204]. 


\section{Future perspectives}

Nanoemulsions are proposed for numerous applications in pharmacy as drug delivery systems because of their capacity to solubilize non-polar active compounds. Future perspectives of nanoemulsion are very promising in different fields of therapeutics or application in development of cosmetics for hair or skin. One of the versatile applications of nanoemulsions is in the area of drug delivery where they act as efficient carriers for bioactives, facilitating administration by various routes. The advantages and applications of nanoemulsions for oral drug delivery are numerous, where the droplet size is related to their absorption in the gastrointestinal tract. Due to the renewed interest in herbal drug formulation, nanoemulsion may be the ideal delivery platform for these difficult-to-formulate phytopharmaceuticals. The prospects of nanoemulsions lie in the ingenuity of formulation experts to utilize the advantages of nanoemulsion carriers in overcoming peculiar problems of drug delivery such as absorption, permeation and stability of both orthodox and herbal drugs.

\section{Conclusion}

Nanoemulsions offer several advantages for the delivery of drugs and are thus receiving increasing attention as drug carriers for improving the delivery of active pharmaceutical ingredients. They are applicable for almost all routes of delivery and therefore hold promise for different fields, be it cosmetics, therapeutics or biotechnology. This new technology could be developed to overcome the poor absorption of some phytopharmaceuticals and poor miscibility of these compounds with the lipid contents of cell membrane linings.

\section{Author details}

S.A. Chime ${ }^{1}$, F.C. Kenechukwu ${ }^{2}$ and A.A. Attama ${ }^{2 *}$

*Address all correspondence to: anthony.attama@unn.edu.ng

1 Department of Pharmaceutical Technology and Industrial Pharmacy, Faculty of Pharmaceutical Sciences, University of Nigeria, Nsukka, Enugu State, Nigeria

2 Drug Delivery Research Unit, Department of Pharmaceutics, Faculty of Pharmaceutical Sciences, University of Nigeria, Nsukka, Enugu State, Nigeria

\section{References}

[1] Ravi T P U, Padma T (2011) Nanoemulsions for drug delivery through different routes. Res. Biotechnol. 2(3): 1-13. 
[2] Kotta S, Khan AW, Pramod K, Ansari S H, Sharma R K, Ali J (2012) Exploring oral nanoemulsions for bioavailability enhancement of poorly water-soluble drugs. Expert Opin. Drug Deliv. 9(5): 585-598.

[3] Dixit RP, Nagarsenker MS (2008) Self-nanoemulsifying granules of ezetimibe: design, optimization and evaluation. Eur. J. Pharm. Sci. 35: 3183-3192.

[4] Shafiq S, Shakeel F, Talegaonkar S (2007) Development and bioavailability assessment of ramipril nanoemulsion formulation. Eur. J. Pharm. Biopharm. 66: 2227-2243.

[5] Sutradhar K B, Amin M L (2013) Nanoemulsions: increasing possibilities in drug delivery. Eur. J. Nanomed. 5(2): 97-110.

[6] Hamed AC, Vitthal VC, Pravin DC (2013) Self emulsifying drug delivery system: A review. Int. J. Pharm. Chem. Sci. 2(1): $34-44$.

[7] Chudasama A, Patel V, Nivsarkar M, Vasu K, Shishoo C (2011) A novel lipid-based oral drug delivery system of nevirapine. Int. J. Pharm. Tech. Res. 3(2):1159-1168.

[8] Jingling T, Jin S, Fude C, Zhonggui H (2006) Preparation of self-emulsifying drug delivery systems of Ginkgo biloba extracts and in vitro dissolution studies. Asian J. Trad. Med. 1: 3-4.

[9] Chime SA, Onyishi VI (2013) Lipid-based drug delivery systems (LDDS): Recent advances and applications of lipids in drug delivery. Afr. J. Pharm. Pharmacol. 7(8): 3034-3059.

[10] Reiss H (1975) Entropy-induced dispersion of bulk liquids. J. Colloid Interf. Sci. 53(1): 61-70.

[11] Gajendra S, Khinchi MP, Gupta MK, Dilip A, Adil H, Natasha S (2012) Self emulsifying drug delivery systems (SEEDS): An approach for delivery of poorly water soluble drug. Int. J. Pharm. Life Sci. 3(9): 991-1996.

[12] Constantinides PP (1995) Lipid microemulsions for improving drug dissolution and oral absorption: physical and biopharmaceutical aspects. Pharm. Res. 12(11): 1561-72.

[13] Dabros T, Yeung A, Masliyah J, Czarnecki J (1999) Emulsification through Area Contraction. J. Colloid Interf. Sci. 210(1): 222-4.

[14] Wakerly MG, Pouton CW, Meakin BJ, Morton FS (1986) Self-emulsification of vegetable oil-non-ionic surfactant mixtures. ACS Symp Series. 311: 242-55.

[15] Rang MJ, Miller CA (1999) Spontaneous emulsification of oils containing hydrocarbon, nonionic surfactant, and oleyl alcohol. J. Colloid. Interf. Sci. 209(1): 179-92.

[16] Bhupinder S, Shantanu B, Rishi K, Ramandeep S, Katare OP (2009) Self-emulsifying drug delivery systems (SEDDS): Formulation development, characterization and applications. Crit. Rev. Ther. Drug Carr. Syst. 26(5): 427-521. 
[17] Patel BR, Patel PR, Patel M (2008) Self- emulsifying drug delivery systems. Pharm. Sci. Tech. 7: 1-3.

[18] Rajan BM, Nirav SS (2011) A review: Self emulsifying drug delivery system. Int. J. Pharm. Pharm. Sci. 3(2): 23-28.

[19] Shobhit K, Satish KG, Pramod KS (2012) Self-emulsifying drug delivery systems (sedds) for oral delivery of lipid based formulations - A review. Afr. J. Basic App. Sci. 4 (1): 07-11.

[20] Obitte NC, Chukwu A (2010) The excipient usefulness of Carbosil® and Landolphia owariensis in two oil-based self-emulsifying formulations. Asian J. Pharmaceut. 4(4): 213- 219.

[21] Sachan R, Khatri K, Kasture SB (2010) Self-emulsifying drug delivery system a novel approach for enhancement of bioavalibility. Int. J. Pharm. Tech. Res. 2(3): 738-1745.

[22] Balakumar K, Raghavan CV, Selvan NT, Prasad RH, Abdu S (2013) Self nanoemulsifying drug delivery system (SNEDDS) of rosuvastatin calcium: Design, formulation, bioavailability and pharmacokinetic evaluation. Colloids Surf. Biointer. 112: 337-43.

[23] Jyoti W, Anroop N, Rachna K (2012) Emulsion forming drug delivery system for lipophilic drugs, Acta Pol. Pharm. Drug Res. 69(2): 179 - 191.

[24] Newton M, Petersson J, Podczeck F, Clarke A, Booth S (2001) The influence of formulation variables on the properties of pellets containing a self-emulsifying mixture. J. Pharm. Sci. 90(8): 987-95.

[25] Gao P, Morozowich W (2006) Development of supersaturatable self-emulsifying drug delivery system formulations for improving the oral absorption of poorly soluble drugs. Expert Opin. Drug Deliv. 3(1): 97-110.

[26] Dahan A, Hoffman A (2008) Rationalizing the selection of oral lipid based drug delivery systems by an in vitro dynamic lipolysis model for improved oral bioavailability of poorly water soluble drugs. J. Control. Rel. 129(1): 1-10.

[27] Morozowich W, Gao P, Charton M (2006) Speeding the development of poorly soluble/poorly permeable drugs by SEDDS/S-SEDDS formulations and prodrugs, Part 1. Am. Pharm. Rev. 9: 110-4.

[28] Gao P, Charton M, Morozowich W (2006) Speeding the development of poorly soluble/poorly permeable drugs by SEDDS/S-SEDDS formulations and prodrugs, Part 2. Am. Pharm. Rev. 9: 16-23.

[29] Tang B, Cheng G, Chun Gu J, Xu Cai-Hong (2008) Development of solid self emulsifying drug delivery system: Preparation techniques and dosage forms. Drug Discovery Today. 13(13/14):606-612.

[30] Kyatanwas AU, Gajdhiye ND, Jadhav KR, Kadam VJ (2010) Solid self emulsifying drug delivery system: A review. J. Pharm. Res. 3(4): 877-882. 
[31] Wadhwa J, Nair A, Kumria R (2012) Emulsion forming drug delivery system for lipophilic drugs. Acta Poloniae Pharmaceutica - Drug Res. 69(2): 179-191.

[32] Shaji J, Jadhav D (2010) Newer approaches to self-emulsifying drug delivery system. Int. J. Pharm. Pharm. Sci. 2(1): 37-42.

[33] Mehta PP, Makanikar V, Parekh PP (2011) Self emulsifying drug delivery system: A novel approach to enhance oral bioavailability of poorly soluble drugs. J. Pharm. Res. 4(7): 2191-2194.

[34] Kumar A, Sharma S, Kamble R (2010) Self emulsifying drug delivery system (SEDDS): Future aspects. Int. J. Pharm. Pharm. Sci. 2(4): 7-13.

[35] Sharma V, Saxena PS, Singh P (2011) Self emulsifying drug delivery system. J. Pharm. Res. 3: 500-504.

[36] Hiral AM, Ami YB, Ramesh BP, Jalpa SP, Tank HM (2013) Self nano emulsifying drug delivery system (SNEDDS): Future aspects. Asian J. Pharm. Res. 2013;3(1): 21-27.

[37] Udaya SM., Josephine RLF, Kiran BU (2013) Self nano emulsifying drug delivery systems for oral delivery of hydrophobic drugs. Biomed. Pharmacol. J. 6(2): 355-362.

[38] Obitte NC, Ofokansi KC, Nzekwe IT, Esimone CO, Okoye IE (2011) Self-nanoemulsifying drug delivery systems based on melon oil and its admixture with a homolipid from Bos indicus for the delivery of indomethacin. Trop. J. Pharm. Res. 10(3): 299-307.

[39] Thomas N, Holm R, Garmer M, Karlsson JJ, Müllertz A, Rades T (2013) Supersaturated self-nanoemulsifying drug delivery systems (Super-SNEDDS) enhance the bioavailability of the poorly water-soluble drug simvastatin in dogs. AAPS PharmSciTech. 15(1):219-227.

[40] Beg S, Swain S, Singh H.P, Patra CN, Rao MB (2013) Development, optimization, and characterization of solid self-nanoemulsifying drug delivery systems of valsartan using porous carriers. AAPS PharmSciTech. 13(4): 1416-1427.

[41] Beg S, Jena SS, Patra CN, Rizwan M, Swain S, Sruti J, Rao MEB, Singh B (2013) Development of solid self-nanoemulsifying granules (SSNEGs) of ondansteron hydrochloride with enhanced bioavailability potential. Colloids Surf. B: Biointer. 101: 414-423.

[42] Zhang Q, He N, Zhang L, Zhu F, Chen Q, Qin Y, Zhang Z, Zhang Q, Wang S (2012) The in vitro and in vivo study on self-nanoemulsifying drug delivery system (SNEDDS) based on insulin-phospholipid complex. J. Biomed. Nanotech. 8 (1): 90-97.

[43] Elsheikh MA, Elnaggar YSR, Gohar EY, Abdallah OY (2012) Nanoemulsion liquid preconcentrates for raloxifene hydrochloride: Optimization and in vivo appraisal. Int. J. Nanomed. 7: 3787-3802. 
[44] Lei Y, Qi J, Nie S, Hu F, Pan W, Lu Y, Wu W (2012) Solid self-nanoemulsifying cyclosporine a pellets prepared by fluid-bed coating: Stability and bioavailability study. J. Biomed. Nanotech. 8(3): 515-521.

[45] Sun M, Han J, Guo X, Li Z, Yang J, Zhang Y, Zhang D (2011) Design, preparation and in vitro evaluation of paclitaxel-loaded self-nanoemulsifying drug delivery system. Asian J. Pharm. Sci. 6(1): 18-25.

[46] Jeevana JB, Sreelakshmi K (2011) Design and evaluation of self-nanoemulsifying drug delivery system of flutamide. J. Young Pharm. 3(1): 4-8.

[47] Abhijit AD, Neha D, Rahul D, Mangal N (2010) Self nanoemulsifying drug delivery systems: Formulation insights, applications and advances. Nanomedicine. 5(10): 1595-1616.

[48] Anjan KM, Narasimha MP, Swadeep B, Ranjit PS (2014) Self-emulsifying drug delivery systems (SEDDS): an update from formulation development to therapeutic strategies. Int. J. PharmTech. Res. 6(2): 546-568.

[49] Cole ET (2008) Challenges and opportunities in the encapsulation of liquid and semisolid formulations into capsules for oral administration. Adv. Drug Deliv. Rev. 60: 747-756.

[50] Rodriguez L, Passerini N, Cavallari C, Cini M, Sancin P, Fini A (1999) Description and preliminary evaluation of a new ultrasonic atomizer for spray-congealing process. Int. J. Pharm. 183:133-143.

[51] Ito Y, Kusawake T, Ishida M, Tawa R (2005) Oral solid gentamicin preparation using emulsifier and adsorbent. J. Control. Rel. 105: 23-31.

[52] Venkatesan N, Yoshimitsu J, Ito Y, Shibata N, Takada K (2005) Liquid filled nanoparticles as a drug delivery tool for protein therapeutics. Biomaterials. 26: 7154-7163.

[53] Venkatesan N, Yoshimitsu J, Ohashi Y, Ito Y, Sugioka N, Shibata N, Takada K (2006) Pharmacokinetic and pharmacodynamic studies following oral administration of erythropoietin mucoadhesive tablets to beagle dogs. Int. J. Pharm. 310: 46-52.

[54] Verreck G, Brewster ME (2004) Melt extrusion-based dosage forms: excipients and processing conditions for pharmaceutical formulations. Bull. Tech. Gattefossé. 24: 8595.

[55] Breitenbach J (2002) Melt extrusion: from process to drug delivery technology. Eur. J. Pharm. Biopharm. 54: 107-117.

[56] Rutvij JP, Gunjan JP, Bharadia PD, Pandya VM, Modi DA (2011) Nanoemulsion: An advanced concept of dosage form. Int. J. Pharm. Cosmetol. 1(5): 122-133.

[57] Yashpal S, Tanuj H, Harsh K (2013) Nanoemulsions: A pharmaceutical review. Int. J. Pharma. Prof. Res. 4(2): 928-935. 
[58] Charles L, Attama AA (2011) Current state of nanoemulsions in drug delivery. J. Biomat. Nanobiotech. 2: 626-639.

[59] Adnan A, Mohammad R, Farhan JA, Zeenat I, Roop KK, Aqil M, Sushama T (2009) Nanoemulsion components screening and selection: a technical note. AAPS PharmSciTech. 10(1): 69-76.

[60] Lawrence MJ, Rees GD (2000) Microemulsion-based media as novel drug delivery systems. Adv. Drug Deliv. Rev. 45: 89-121.

[61] Narang AS, Delmarre D, Gao D (2007) Stable drug encapsulation in micelles and microemulsions. Int. J. Pharm. 345:9-25.

[62] Constantinides PP (1995) Lipid microemulsions for improving drug dissolution and oral absorption and biopharmaceutical aspects. Pharm. Res. 12: 1561-1572.

[63] Karim A, Gokhale R, Cole M, Sherman J, Yeramian P, Bryant M, Franke H (1994) HIV protease inhibitor SC-52151: a novel method of optimizing bioavailability profile via a microemulsion drug delivery system. Pharm. Res. 11:S368.

[64] Kawakami K, Yoshikawa T, Moroto Y, Kanaoka E, Takahashi K, Nishihara Y, Masuda K (2002) Microemulsion formulation for enhanced absorption of poorly soluble drugs. II. In vivo study. J. Control. Rel. 81:75-82.

[65] Kommuru TR, Gurley B, Khan MA, Reddy IK (2001) Self-emulsifying drug delivery systems (SEDDS) of coenzyme Q10: formulation development and bioavailability assessment. Int. J. Pharm. 212: 233-246.

[66] Kreilgaard M, Pedersen EJ, Jaroszewski JW (2000) NMR characterization and transdermal drug delivery potential of microemulsion systems. J. Control. Rel. 69: 421433.

[67] Tenjarla S (1999) Microemulsions: an overview and pharmaceutical applications. Crit. Rev. Ther. Drug Carrier Syst. 16: 461-521.

[68] Attwood D (1994) Microemulsions. In: Kreuer J, Editor. Colloidal drug delivery systems. New York: Marcel Dekker. pp. 31-71.

[69] Ravi TPU, Padma T (2011) Nanoemulsions for drug delivery through different routes. Research in Biotechnol. 2(3): 1-13.

[70] Anton N, Vandamme T (2009) The universality of low-energy nano-emulsification. Int. J. Pharm. 377 (1-2): 142-147.

[71] Mason TG, Graves SM, Wilking JN, Lin MY (2006) Extreme emulsification: formation and structure of nanoemulsions. J. Phy. Cond. Matter. 9(1): 193-199.

[72] Tadros T, Izquierdo P, Esquena J, Solans C (2004) Formation and stability of nanoemulsions. Adv. Colloid Interface Sci. 108-109: 303-318.

[73] Lamaallam S, Bataller H, Dicharry C, Lachaise J (2005) Formation and stability of mini-emulsions produced by dispersion of water/oil/surfactants concentrates in a 
large amount of water. Colloid. Surf. A: Physicochem. Eng. Aspects. 270-271(1-3): 44-51.

[74] Solans C, Esquena J, Forgiarini AM, Uson N, Morales D, Izquierdo P (2002) Nanoemulsions: formation and properties. In: Mittal KL, Shah DO, Editors. Surfactants in solution: fundamentals and applications. New York: Marcel Dekker. pp. 525.

[75] Izquierdo P, Feng J, Esquena J, Tadros TF, Dederen JC, Garcia MJ (2005) The influence of surfactant mixing ratio on nano-emulsion formation by the pit method. J. Colloid Interf. Sci. 285(1): 388-394.

[76] Shinoda K, Saito H (1968) The effect of temperature on the phase equilibria and the types of dispersions of the ternary system composed of water, cyclohexane, and nonionic surfactant. J. Colloid Interf. Sci. 26(1): 70-74.

[77] Forgiarini A, Esquena J, Gonzalez C, Solans C (2001) Formation of nano-emulsions by low-energy emulsification methods at constant temperature. Langmuir. 17: 20762083.

[78] Pey CM, Maestro A, Solé I, González C, Solans C, Gutiérrez JM (2006) Optimization of nano-emulsions prepared by low-energy emulsification methods at constant temperature using a factorial design study. Colloids Surf. A: Physicochem. Eng Aspects. 288(1-3): 144-150.

[79] Porras M, Solans C, González C, Gutiérrez JM (2008) Properties of water-in-oil (W/O) nano-emulsions prepared by a low-energy emulsification method. Colloids Surf. A: Physicochem. Eng. Aspects. 324(1-3): 181-188.

[80] Sonneville-Aubrun O, Simonnet JT, L'Alloret F (2004) Nanoemulsions: A new vehicle for skincare products. Adv. Colloids Interf. Sci. 108-109: 145-149.

[81] Solans C, Izquierdo P, Nolla J, Azemar N, Garcia-Celma MJ (2005) Nano-emulsions. Curr. Opin. Colloid Interf. Sci. 10 (3-4): 102-110.

[82] Wang L, Li X, Zhang G, Dong J, Eastoe J (2007) Oil-in-water nanoemulsions for pesticide formulations. J. Colloid Interf. Sci. 314(1): 230-235.

[83] Sole I, Maestro A, Pey CM, Gonzalez C, Solans C, Gutierrez JM (2006) Nanoemulsions preparation by low energy methods in an ionic surfactant system. Colloids Surf. A: Physiochem. Eng. Aspects. 288: 138-143.

[84] Sole I, Pey CM, Maestro A, Gonzalez C, Porras M, Solans C, Gutierrez JM (2010) Nanoemulsions prepared by phase inversion composition method: preparation variables and scale up. J. Colloid Interf. Sci. 344(2): 417-423.

[85] Graves S, Meleson K, Wilking J (2005) Structure of concentrated nanoemulsions. J. Chem. Phys. 122 (13): Article ID 134703. doi:10.1063/1.1874952

[86] Jafari SM, He Y, Bhandari B (2007) Optimization of nanoemulsion production by microfluidization. Eur. Food Res. Tech. 225 (5-6): 733-741. 
[87] Quin C, Mc Clement DJ (2011) Formation of nanoemulsions stabilized by model food grade emulsifiers using high pressure homogenization: factors affecting particle size. Food Hydrocolloids. 25(5): 1000-1008.

[88] Shafiq S, Faiyaz S, Sushma T, Ahmad FJ, Khar RK, Ali M (2007) Development and bioavailability assessment of ramipril nanoemulsion formulation. Eur. J. Pharm. Biopharm. 66(2): 227-243.

[89] Shakeel F, Baboota S, Ahuja A, Ali J, Faisal MS, Shafiq S (2008) Stability evaluation of celecoxib nanoemulsion containing Tween 80. Thailand J. Pharm. Sci. 32: 4-9.

[90] Shakeel F, Baboota S, Ahuja A, Ali J, Shafiq S (2008) Skin permeation mechanism of aceclofenac using novel nanoemulsion formulation. Pharmazie. 63(8): 580-584.

[91] Baboota S, Shakeel F, Ahuja A, Ali J, Shafiq S (2007) Design, development and evaluation of novel nanoemulsion formulations for transdermal potential of celecoxib. Acta Pharm. 57: 316- 332.

[92] Mason TG, Wilking JN, Meleson K, Chang CB, Graves SM (2006) Nanoemulsions: formation, structure and physical properties. J. Phys-Condens. Matter. 18:636-643.

[93] Haritha A, Syed PB, Koteswara RP, Chakravarthi V (2013) A brief introduction to methods of preparation, applications and characterization of nanoemulsion drug delivery systems. Indian J. Res. Pharm. Biotechnol. 1 (1): 25-28.

[94] Narang AS, Delmarre D, Gao D (2007) Stable drug encapsulation in micelles and microemulsions. Int. J. Pharm. 345: 9-25.

[95] Gursoy RN, Benita S (2004) Self-emulsifying drug delivery systems (SEDDS) for improved oral delivery of lipophilic drugs. Biomed. Pharmacotherap. 58: 173-82.

[96] Gershanik T, Benzeno S, Benita S (1998) Interaction of the self-emulsifying lipid drug delivery system with mucosa of everted rat intestine as a function of surface charge and droplet size. Pharm. Res. 15: 863-9.

[97] Ghosh PK, Majithiya RJ, Umrethia ML, Murthy RSR (2006) Design and development of microemulsion drug delivery system of acyclovir for improvement of oral bioavailability. AAPS PharmSciTech. 7(3): Article 77.

[98] Lawrence MJ, Rees GD (2000) Microemulsion-based media as novel drug delivery systems. Adv. Drug Deliv. Rev. 45: 89-121.

[99] Chiesa M, Garg J, Kang YT, Chen G (2008) Thermal conductivity and viscosity of water in oil nanoemulsions. Colloid Surf. A. 326: 67-72.

[100] Craig DQM, Barker SA, Banning D, Booth SW (1995) An investigation into the mechanisms of self-emulsification using particle size analysis and low frequency dielectric spectroscopy. Int. J. Pharm. 114:103-10. 
[101] Debnath S, Satayanarayana P, Gampa VK (2011) Nanoemulsion- a method to improve the solubility of lipophilic drugs. PHARMANEST - Int. J. Adv. Pharm. Sci. 2(2-3): 72-83.

[102] Tin-hinan K, Elmira A-T, Nabila B, Michel L (2011) Physico-chemical characterization of nanoemulsions in cosmetic matrix enriched on omega-3. J. Nanobiotechnol. 9:41, $1-8$.

[103] Karthikeyan S, Jeeva PA, Jerobin J, Mukherjee A, Chandrasekaran N (2012) Formulation and characterization of nanoemulsion coatings from Azadirachta indica. Int. J. CheTech. Res. 4(4): 566-1570.

[104] Mandal A, Bera A (2012) Surfactant stabilized nanoemulsion: Characterization and application in enhanced oil recovery. World Acad Sci. Eng. Tech. 67: 21-26.

[105] Kayes JB (1999) Disperse systems. In: Aulton ME, Editor. Pharmaceutics The Science of Dosage Form Design. Edinburgh: Churchill Livingstone. pp. 81-118, 571.

[106] Barea MJ, Jekins MJ, Gaber MH (2010) Evaluation of liposomes coated with a pH responsive polymer. Int. J. Pharm. 402(1): 89-94.

[107] Samah NA, Williams N, Heard CM (2010) Nanogel particulates located within diffusion cell receptor phases following topical application demonstrates up-take into and migration across skin. Int. J. Pharm. 401(1-2): 72-78.

[108] Ruth HS, Attwood D, Ktistis G, Taylor C (1995) Phase studies and particle size analysis of oil-in-water phospholipid microemulsions. Int. J. Pharm. 116 (2): 253-261.

[109] Li X, Anton N, Ta TMC, Zhao M, Messaddeq N, Vandamme TF (2011) Microencapsulation of nano-emulsions: Novel trojan particles for bioactive lipid molecule delivery. Int. J. Nanomed. 6: 1313-1325.

[110] Agrawal Y, Petkar KC, Sawant KK (2010) Development, evaluation and clinical studies of acitretin loaded nanostructured lipid carriers for topical treatment of psoriasis. Int. J. Pharm. 401(1-2): 93-102.

[111] Touitou E, Dayan N, Bergelson L (2000) Ethosomes - Novel vesicular carriers for enhanced delivery: Characterization and skin penetration properties. J. Control. Rel. 65 (3): 403-418.

[112] Bendas ER, Tadros MI (2007) Enhanced transdermal delivery of salbutamol sulfate via ethosomes. AAPS PharmSciTech. 8(4): 214-220.

[113] Jain S, Tiwary AK, Sapra B (2007) Formulation and evaluation of ethosomes for transdermal delivery of lamivudine. AAPS PharmSciTech. 8(4): 249-257.

[114] Dave V, Kumar D, Lewis S (2010) Ethosome for enhanced transdermal drug delivery of aceclofenac. Int. J. Drug Deliv. 2: 81-92. 
[115] Subhashis D, Satayanarayana J, Gampa VK (2011) Nanoemulsion-a method to improve the solubility of lipophilic drugs. PHARMANEST- Int. J. Adv. Pharm. Sci. 2(2-3): 72-83.

[116] Surbhi S, Kumkum S (2012) Nanoemulsions for cosmetics. Int. J. Adv. Res. Pharma. Bio. Sci. 2(3): 408-415.

[117] Hughes PM, Mitra AK (1993) Overview of ocular drug delivery and iatrogenic ocular cytopathologies. In: Mitra AK, Editor. Ophthalmic drug delivery systems. New York: Marcel Dekker. p. 1-27.

[118] Patton TF, Robinson JR (1976) Quantitative precorneal disposition of topically applied pilocarpine nitrate in rabbit eyes. J. Pharm. Sci. 65: 1295-301.

[119] Sieg JW, Robinson JR (1977) Vehicle effects on ocular drug bioavailability II: evaluation of pilocarpine. J. Pharm. Sci. 66: 1222-8.

[120] Chein YW, Cabana BE, Mares SE (1982) Ocular controlled release drug administration. In: Chein YW, Eitor. Novel drug delivery systems; fundamentals, development concepts, biomedical assessments (drugs and the pharmaceutical sciences). New York: Marcel Dekker. p. 13-55.

[121] Middleton DL, Leung SS, Robinson JR (1990) Ocular bioadhesive delivery systems. In: Lenaerts V, Gurny R, Editors. Bioadhesive drug delivery systems. Boca Raton: CRC. p. 179-202.

[122] Hussein OA, Salama HA, Ghorab M, Mahmoud AA (2009) Nanoemulsion as a potential ophthalmic delivery system for dorzolamide hydrochloride. AAPS PharmSciTech. 10(3): 808-819.

[123] Muller-Goymann CC (2004) Physicochemical characterization of colloidal drug delivery systems such as reverse micelles, vesicles, liquid crystals and nanoparticles for topical administration. Eur J Pharm Biopharm. 58(2): 343-356.

[124] Gaur PK, Mishra S, Purohit S, Dave K (2009) Trans-dermal drug delivery system: A review. Asian J. Pharm. Clin. Res. 2(1): 14-20.

[125] Shakee F, Ramadan W (2010) Transdermal delivery of anticancer drug caffeine from water-in-oil nanoemulsions. Colloids Surf. B: Biointer. 75(1): 356-362.

[126] Osborne DW, Ward AJ, Neil KJ (1991) Microemulsions as topical delivery vehicles: In vitro transdermal studies of a model hydrophilic drug. J. Pharm. Pharmacol. 43 (6): 450- 454 .

[127] Trotta M, Pattarino F, Gasco MR (1996) Influence of counter ions on the skin permeation of methotrexate from water-oil microemulsions. Pharmaceutia Acta Helvetiae. 71 (2): 135-140. 
[128] Delgado-Charro MB, Iglesias-Vilas G, Blanco- Mendez J, Lopez-Quintela MJ, Marty MA, Guy JP (1997) Delivery of a hydrophilic solute through the skin from novel microemulsion systems. Eur. J. Pharm. Biopharm. 43(1): 37-42.

[129] Dreher F, Walde P, Walter P, Wehrli E (1997) Interaction of a lecithin microemulsion gel with human stratum corneum and its effect on transdermal transport. J. Control. Rel. 45 (2):131-140.

[130] Schmalfus U, Neubart R, Wohlrab W (1997) Modification of drug penetration into human skin using microemulsions. J. Control. Rel. 46(3): 279-285.

[131] Alvarez-Figueroa MJ, Blanco-Mendez J (2001) Trans-dermal delivery of methotrexate: Iontophoretic delivery from hydrogels and passive delivery from microemulsions. Int. J. Pharm. 215(1-2): 57-65.

[132] Rhee YS, Choi JG, Park ES, Chi SC (2001) Trans-dermal delivery of ketoprofen using microemulsions. Int. J. Pharm. 228(1): 161-170.

[133] Lee PJ, Langer R, Shastri VP (2003) Novel microemulsion enhancer formulation for simultaneous trans-dermal delivery of hydrophilic and hydrophobic drugs. Pharm. Res. 20(2): 264-269.

[134] Kemken JA, Ziegler A, Muller BW (1992) Influence of supersaturation on the pharmacodynamic effect of bupranolol after dermal administration using microemulsions as vehicle. Pharm. Res. 9(4): 554-558.

[135] Kreilgaard M (2001) Dermal pharmacokinetics of microemulsion formulations determined by in-vitro microdialysis. Pharm. Res. 18(3): 367-373.

[136] Kreilgaard M, Kemme MJB, Burggraaf J, Schoemaker RC, Cohen AF (2001) Influence of a microemulsion vehicle on cutaneous bioequivalence of a lipophilic model drug assessed by microdialysis and pharmacodynamics. Pharm. Res. 18(5): 593-599.

[137] Ktistis G, Niopas I (1998) A study on the in-vitro percutaneous absorption of propranolol from disperse systems. J. Pharm. Pharmacol. 50: 413-419.

[138] Gasco MR, Gallarate M, Pattarino F (1999) In vitro permeation of azelaic acid from viscosized microemulsions. Int. J. Pharm. 69: 193-196.

[139] Kriwet K, Muller-Goymann CC (1995) Diclofenac release from phospholipid drug systems and permeation through excised human stratum corneum. Int. J. Pharm. 125(2): 231-242.

[140] Trotta M (1999) Influence of phase transformation on indomethacin release from microemulsions. J. Control. Rel. 60(2): 399-405.

[141] Sheela AY, Sushil KP, Singh DK, Shaikh A (2012) A review: Nanoemulsion as vehicle for transdermal permeation of antihypertensive drug. Int. J. Pharm. Pharm. Sci. 4(1): 41-44. 
[142] Barakat N, Fouad E, Elmedany A (2011) Formulation design of indomethacin-loaded nanoemulsion for transdermal delivery. Pharm. Anal. Acta S2:002. doi: 10.4172/2153-2435.S2-002

[143] Singh BP, Kumar B, Jain SK, Shafaat K (2012) Development and characterization of a nanoemulsion gel formulation for transdermal delivery of carvedilol. Int. J. Drug Dev. Res. 4(1): 151-161.

[144] Sajid A, Sarfaraz A, Nawazish A, Syed ASQ, Intakhab A, Shamim P, Daud A (2013) Formulation, characterization and in vivo assessment of topical nanoemulsion of betamethasone valerate for psoriasis and dermatose. Int. J. Pharm. 3(1): 186-199.

[145] Huafeng Z, Yang Y, Guanlan L, Yan L, Jing Z, Qiu G, Zemin Y, Mingxing D (2010) Preparation and characterization of a lecithin nanoemulsion as a topical delivery system. Nanoscale Res. Lett. 5: 224-230.

[146] Modi JD, Patel JK (2011) Nanoemulsion-based gel formulation of aceclofenac for topical delivery. Int. J. Pharm. Pharm. Sci. Res. 1(1): 6-12.

[147] Ranjit KH, Kartik CP, Surendra KP, Jagadish S, Mohammed AR (2011) Nanoemulsions as vehicles for transdermal delivery of glycyrrhizin. Braz. J. Pharm. Sci. 47(4): 13-19.

[148] Inayat BP, Setty CM (2011) Enhancement of transdermal delivery of tamoxifen citrate using nanoemulsion vehicle. Int. J. PharmTech Res. 3(1): 287-297.

[149] Shakeel F, Sanjula B, Alka A, Javed A, Mohammed A, Sheikh S (2007) Nanoemulsions as vehicles for transdermal delivery of aceclofenac. AAPS PharmSciTech. 8(4): Article 104.

[150] Shakeel F, Sheikh S, Nazrul H, Fars KA, Ibrahim AA (2012) Nanoemulsions as potential vehicles for transdermal and dermal delivery of hydrophobic compounds: an overview. Expert Opin. Drug Deliv. 9(8): 953-974.

[151] Ranjit KH, Kartik CP, Pareta SK (2011) Nanoemulsion as potential vehicles for transdermal delivery of pure phytopharmaceuticals and poorly soluble drug. Int. J. Drug Deliv. 3: 209-218.

[152] Primo FL, Michieloto L, Rodrigues MAM, Macaroff PP, Morais PC, Lacava ZGM, Bently MVLB, Tedesco AC (2007) Magnetic nanoemulsions as drug delivery system for Foscan: skin permeation and retention in vitro assays for topical application in photodynamic therapy (PDT) of skin cancer. J. Magnet Magnet. Mat. 311(1): 354-357.

[153] Pires A, Fortuna A, Alves G, Falcao A (2009) Intranasal drug delivery: How, why and what for? J. Pharm. Pharm. Sci. 12: 288-311.

[154] Ugwoke MI, Agu RU, Verbeke N, Kinget R (2005) Nasal mucoadhesive drug delivery: Background, applications, trends, and future perspectives. Adv. Drug Deliv. Rev. 57(11): 1640- 1665. 
[155] Pardridge WM (1999) Non-invasive drug delivery to human brain using endogenous blood brain barrier transport system. Pharm. Sci. Tech. Today. 2(2): 49-59.

[156] Kumar M, Misra A, Babbar AK, Mishra AK (2008) Intranasal nanoemulsion based brain targeting drug delivery system of risperidone. Int. J. Pharm. 358 (1-2): 285-291.

[157] Mistry A, Stolnik S, Illum L (2009) Nanoparticles for direct nose-to-brain delivery of drugs. Int. J. Pharm. 379(1): 146-157.

[158] Csaba N, Garcia-Fuentes M, Alonsa MJ (2009) Nanoparticles for nasal vaccination. Adv. Drug Deliv. Rev. 61(2): 140-157.

[159] Clark AM, Jepson MA, Hirst BH (2001) Exploiting M cells for drug and vaccine delivery. Adv. Drug Deliv. Rev. 50(1-2): 81-106.

[160] Bhanushali RS, Gatne MM, Gaikwad RV, Bajaj AN, Morde MA (2009) Nanoemulsion based intranasal delivery of anti-migraine drugs for nose to brain targeting. Indian J. Pharm. Sci. 71(6): 707-709.

[161] Tamilvanan S (2004) Submicron emulsions as a carrier for topical (ocular and percutaneous) and nasal drug delivery. Indian J. Pharm. Educ. 38(2):73-8.

[162] Patel R, Patel KP (2010) Advances in novel parenteral drug delivery systems. Asian J. Pharm. 4(3): 193-199.

[163] Araujo FA, Kelmann RG, Araujo BV, Finatto RB, Teixeira HF, Koester LS (2011) Development and characterization of parenteral nanoemulsions containing thalidomide. Eur. J. Pharm. Sci. 42(5): 238-245.

[164] Ganta S, Deshpande D, Korde A, Amiji M (2010) A review of multifunctional nanoemulsion systems to overcome oral and CNS drug delivery barriers. Mol. Membr. Biol. 27(7): 260-273.

[165] Kelmann RG, Kuminek G, Teixeira H, Koester LS (2007) Carbamazepine parenteral nanoemulsions prepared by spontaneous emulsification process. Int. J. Pharm. 342 (1-2): 231-239.

[166] Brussel F, Manoela L, Luisa BW, Michelle F, Koester LS, Teixeira HF (2012) Nanoemulsions as parenteral drug delivery systems: A review. Chem New. 35 (9):34-39.

[167] Gleeson D, Rose JD, Smith PM (1983) A prospective randomised controlled trial of diazepam (valium) vs emulsified diazepam (diazemuls) as a premedication for upper gastrointestinal endoscopy. Br. J. Clinical Pharm. 16: 448.

[168] von Dardel O, Mebius C, Mossberg T, Svensson B (1983) Fat emulsion as a vehicle for diazepam, a study in 9492 patients. Br. J. Anaesthesia. 55: 41.

[169] Doenicke AW, Roizen MF, Hoernecke R, Lorenz W, Ostwald P (1999) Solvent for etomidate may cause pain and adverse effects. Br. J. Anaesthesia. 83: 464 - 466. 
[170] Nyman Y, von Hofsten K, Palm C, Eksborg S, Lonnqvist PA (2006) Etomidate-Lipuro is associated with considerably less injection pain in children compared with propofol with added lidocaine. Br. J. Anaesthesia. 97: 536-539.

[171] Doenicke AW, Roizen MF, Rau J, O'Connor M, Kugler J, Klotz U (1997) Pharmacokinetics and pharmacodynamics of propofol in a new solvent. Babl. J. Anesthesia \& Analgesia. 85: 1399-1403.

[172] Nebauer AE, Doenicke A, Hoernecke R, Angster R, Mayer M (1992) Does etomidate cause haemolysis? Br. J. Anaesthesia. 69 (1):58-60.

[173] Doenicke A, Kugler A, Vollmann N (1990) Venous tolerance to etomidate in lipid emulsion or propylene glycol (hypnomidate). Can. J. Anesthesia. 37(7): 823-4.

[174] Calvo R, Telletxea S, Leal N, Aguilera L, Suarez E, De La Fuente L, Martin-Suarez A, Lukas JC (2004) Influence of formulation on propofol pharmacokinetics and pharmacodynamics in anesthetized patients. Acta Anaesthesiologica Scandinavica. 48 (8): 1038-1048.

[175] http://www.accessdata.fda.gov/scripts/cder/drugsatfda/index.cfm, acessada em Junho 2012.

[176] http://www7.anvisa.gov.br/datavisa/consulta_produto/Medicamentos/frmConsultaMedicamentos.asp, acessada em Junho 2012.

[177] Schicher M, Polsinger M, Hermetter A, Prassl R, Zimmer A (2008) In vitro release of propofol and binding capacity with regard to plasma constituents. Eur. J. Pharm. Biopharm. 70 (3): 882-8.

[178] Ward DS, Norton JR, Guivarc'h PH, Litman RS, Bailey PL (2002) Pharmacodynamics and pharmacokinetics of propofol in a medium-chain triglyceride emulsion. Anesthesiology. 97 (6):1401-1408.

[179] Rau J, Roizen MF, Doenicke AW, O'Connor MF, Strohschneider U (2001) Propofol in an emulsion of long- and medium-chain triglycerides: the effect on pain. Anesthesia \& Analgesia. 93: 382-384.

[180] Knibbe CA, Voortman HJ, Aarts LP, Kuks PF, Lange R, Langemeijer HJ, Danhof M (1999) Pharmacokinetics, induction of anaesthesia and safety characteristics of propofol 6\% SAZN vs propofol 1\% SAZN and Diprivan-10 after bolus injection. Br. J. Clin. Pharm. 47(6): 653-60.

[181] Cox EH, Knibbe CA, Koster VS, Langemeijer MW, Tukker EE, Lange R, Kuks PF, Langemeijer HJ, Lie AHL, Danhof M (1998) Influence of different fat emulsion-based intravenous formulations on the pharmacokinetics and pharmacodynamics of propofol. Pharm. Res. 15 (3): 442-8. 
[182] Knibbe CA, Naber H, Aarts LP, Kuks PF, Danhof M (2004) Long-term sedation with propofol $60 \mathrm{mg} \mathrm{ml-1}$ vs propofol $10 \mathrm{mg}$ ml-1 in critically ill, mechanically ventilated patients. Acta Anaesthesiologica Scandinavica. 48(3): 302-307.

[183] Fechner J, Schwilden H, Schuttler J (2008) Handbook of Experimental Pharmacology. falta editora: falta cidade. p. 253.

[184] Panyam J, Labhasetwar V (2004) Sustained cytoplasmic delivery of drugs with intracellular receptors using biodegradable nanoparticles. Mol. Pharmacol. 1(1): 77 - 84.

[185] Yokoyama K, Watanabe M (1996) Limethason as a lipid microsphere preparation. Adv. Drug Delivery Rev. 20: 195-201.

[186] Kumpulainen E, Valitalo P, Kokki M, Lehtonen M, Hooker A, Ranta VP, Kokki H (2010) Plasma and cerebrospinal fluid pharmacokinetics of flurbiprofen in children. Br. J. Clin. Pharm. 70: 557 - 566.

[187] Hoshi K (1996) Approved indications of lipo-PGE1 in Japan. Adv. Drug Delivery Rev. 20: 171 - 176.

[188] Igarashi R, Takenaga M, Takeuchi J, Kitagawa A, Matsumoto K, Mizushima Y (2001) Marked hypotensive and blood flow-increasing effects of a new lipo-PGE(1) (lipoAS013) due to vascular wall targeting. J. Control. Rel. 71(2): 157-164.

[189] Momma K (1996) Lipo-PGE1 treatment of the neonate with critical congenital heart disease and ductus-arteriosus dependent circulation. Adv. Drug Delivery Rev. 20: 177-180.

[190] Scheffler P, Gross J, Markwirth T, Maier J, Schieffer H (1996) Progress in the prostaglandin E1-therapy of the intermittent claudication by means of bolus injection of LIPO-prostaglandin E1 (LIPO-PGE1). Eur. J. Clin. Pharm. 51(3-4): 235-239.

[191] Heidi MM, Yun-Seok R, Xiao W (2009) Nanomedicine in pulmonary delivery. Int. J. Nanomed. 4: 299-319.

[192] Bivas-Benita M, Oudshoorn M, Romeijn S (2004) Cationic submicron emulsions for pulmonary DNA immunization. J. Control. Rel. 100(1): 145-155.

[193] Lawrence MJ, Rees GD (2000) Microemulsion-based media as novel drug delivery systems. Adv. Drug. Deliv. Rev. 45:89-121.

[194] Liu F, Yang J, Huang L, Liu D (1996) Effect of non- ionic surfactants on the formation of DNA/emulsion complexes and emulsion-mediated gene transfer. Pharm. Res. 13(11):1642-1646.

[195] Yi SW, Yune TY, Kim TW, Chung H (2000) A cationic lipid emulsion/DNA complex as a physically stable and serum-resistant gene delivery system. Pharm. Res. 17(3): 314-320. 
[196] Liu C-H, Yu S-Y (2010) Cationic nanoemulsions as non-viral vectors for plasmid DNA delivery. Colloids Surf. B: Biointer. 79(2)509-515.

[197] André LS, Francisco AJ, Lourena MV, Lucymara F A-L, Lucila CME, Anselmo GO, Eryvaldo STE (2012) Physical factors affecting plasmid DNA compaction in stearylamine-containing nanoemulsions intended for gene delivery. Pharmaceuticals. 5: 643-654.

[198] Saraf S (2010) Applications of novel drug delivery system for herbal formulations. Fitoterapia. 81: 680-689.

[199] Goyal A, Kumar S, Nagpal M, Singh I, Arora S (2011) Potential of novel drug delivery systems for herbal drugs. Indian J. Pharm. Educ. Res. 45: 225.

[200] Uhumwangho M, Okor R (2005) Current trends in the production and biomedical applications of liposomes: A review. J. Med. Biomed. Res. 4: 9-21.

[201] Youenang-Piemi MP, Korner D, Benita S, Marty JP (1999) Positively and negatively charged submicron emulsions for enhanced topical delivery of antifungal drugs. J. Control. Rel. 58: 177-187.

[202] Shen Q, Wang Y, Zhang Y (2011) Improvement of colchicine oral bioavailability by incorporating eugenol in the nanoemulsion as an oil excipient and enhancer. Int. J. Nanomed. 6: 1237 - 1243.

[203] Silva AP, Nunes BR, De Oliveira MC, Koester LS, Mayorga P, Bassani VL, Teixeira HF (2009) Development of topical nanoemulsions containing the isoflavone genistein. Pharmazie. 64: 32-35.

[204] Wang X, Jiang Y, Wang Y-W, Huang M-T, Ho C-T, Huang Q (2008) Enhancing antiinflammation activity of curcumin through $\mathrm{o} / \mathrm{w}$ nanoemulsions. Food Chemistry. 108: 419-424. 